\title{
Synthesis of Sensor Fish Data for Assessment of Fish Passage Conditions at Turbines, Spillways, and Bypass Facilities - Phase 1: The Dalles Dam Spillway Case Study
}

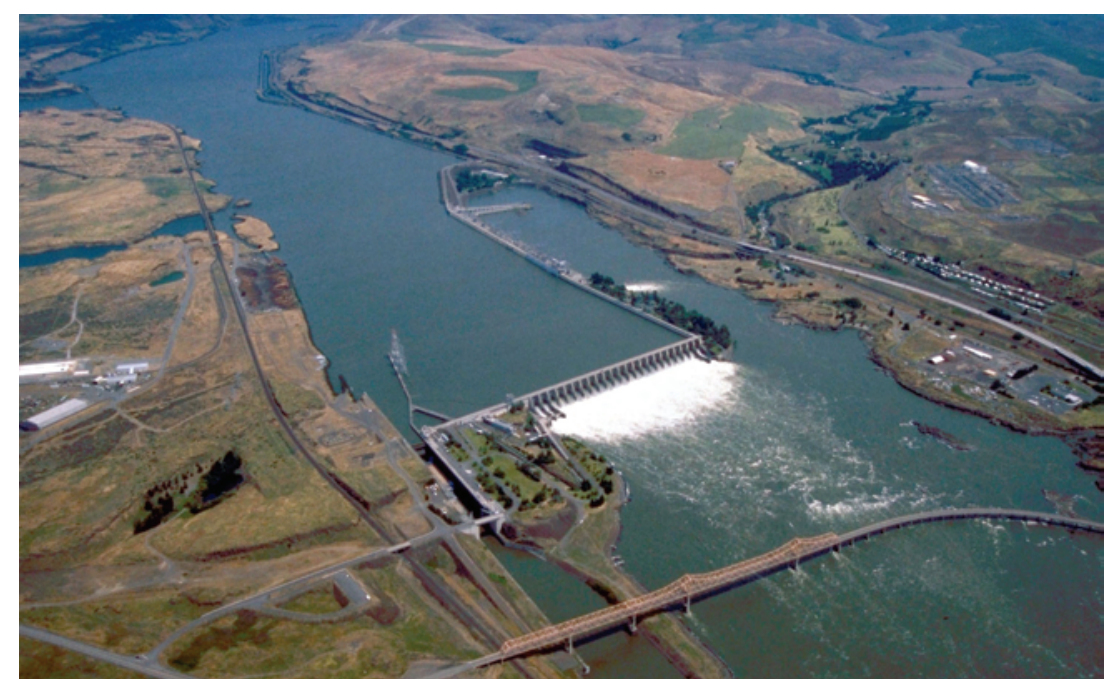

Z. Deng

J. A. Serkowski

T. $\mathrm{Fu}$

T. J. Carlson

M. C. Richmond

Final Report

December 31, 2007

Prepared for the U.S. Army Corps of Engineers

Portland District, Portland, Oregon under a Related Services Agreement with the U.S. Department of Energy Contract DE-AC05-76RL01830 


\title{
DISCLAIMER
}

This report was prepared as an account of work sponsored by an agency of the United States Government. Neither the United States Government nor any agency thereof, nor Battelle Memorial Institute, nor any of their employees, makes any warranty, express or implied, or assumes any legal liability or responsibility for the accuracy, completeness, or usefulness of any information, apparatus, product, or process disclosed, or represents that its use would not infringe privately owned rights. Reference herein to any specific commercial product, process, or service by trade name, trademark, manufacturer, or otherwise does not necessarily constitute or imply its endorsement, recommendation, or favoring by the United States Government or any agency thereof, or Battelle Memorial Institute. The views and opinions of authors expressed herein do not necessarily state or reflect those of the United States Government or any agency thereof.

\author{
PACIFIC NORTHWEST NATIONAL LABORATORY \\ operated by \\ BATTELLE \\ for the \\ UNITED STATES DEPARTMENT OF ENERGY \\ under Contract DE-AC05-76RL01830
}

Printed in the United States of America

Available to DOE and DOE contactors from the

Office of Scientific and Technical Information,

P.O. Box 62, Oak Ridge, TN 37831-0062;

ph: (865) 576-8401

fax: (865) 576-5728

email: reports@adonis.osti.gov

\author{
Available to the public from the National Technical Information Service, \\ U.S. Department of Commerce, 5285 Port Royal Rd., Springfield, VA 22161 \\ ph: (800) 553-6847 \\ fax: (703) 605-6900 \\ email: orders@ntis.fedworld.gov \\ online ordering: http://www.ntis.gov/ordering.htm
}

This document was printed on recycled paper.

$(9 / 2003)$ 


\title{
Synthesis of Sensor Fish Data for Assessment of Fish Passage Conditions at Turbines, Spillways, and Bypass Facilities - Phase 1: The Dalles Dam Spillway Case Study
}

\author{
Z. Deng \\ J. A. Serkowski \\ T. Fu \\ T. J. Carlson \\ M. C. Richmond
}

Final Report

December 31, 2007

Prepared for the U.S. Army Corps of Engineers

Portland District, Portland, Oregon

under a Related Services Agreement

with the U.S. Department of Energy

Contract DE-AC05-76RL01830

Pacific Northwest National Laboratory

Richland, Washington 99352 



\section{Summary}

This report summarizes the characterization of spillway passage conditions at The Dalles Dam in 2006. It also describes the effort to complete a comprehensive database for data sets from experiments with Sensor Fish and balloon-tagged live fish at The Dalles Dam spillway and evaluate the database as an efficient means for accessing and retrieving system-wide data for the U.S Army Corps of Engineers (USACE).

To meet the objective of characterizing the spillway conditions, Sensor Fish devices were released in April 2006 concurrently with balloon-tagged live fish-25 in mid-Bay 4 and 31 in Bay 6 - at the target release elevation of $131 \mathrm{ft}$. In addition, 6 Sensor Fish were deployed upstream of the vortex and another 12 into the surface of the vortex. Release elevations for the mid-bay releases were estimated assuming hydrostatic conditions. Release elevations computed from Sensor Fish pressure measurements indicated that Sensor Fish might have been released deeper in Bay 4 ( 135 ft) than those in Bay 6 ( 138 ft), with the planned release elevation being $131 \mathrm{ft}$. The accuracy of the hydrostatic condition assumption can be evaluated using flow information from computational fluid dynamics (CFD). For high-volume spills at The Dalles, non-hydrostatic conditions exist near the release pipe exits, and the release elevation error assuming hydrostatic conditions can be up to 2 to $3 \mathrm{ft}$. In addition, Sensor Fish released at Bay 4 measured higher peak pressure in the transition region (from spillway chute to tailwater) than Sensor Fish released at Bay 6, possibly because of the deeper releases at Bay 4 and the existence of a high-pressure zone in the lower water column. However, we have no conclusive evidence from which to determine whether the release pipes exits were actually placed at the target elevations or whether the apparent elevation differences were caused by the non-hydrostatic pressure fields that exist and could be different at Bays 4 and 6.

During passage from the entry points to the baffle blocks, 40\% of Sensor Fish released into mid-Bay 4 experienced at least one severe event, while 16\% of Sensor Fish released into mid-Bay 6 had at least one severe event. Overall, for the mid-bay releases in this study, Sensor Fish released from Bay 6 had smoother passage than those released from Bay 4. This finding is consistent with the findings of the live fish study in which fish released from Bay 4 sustained higher injury rates than fish from Bay 6 and the survival rate at Bay 6 was higher than that at Bay 4. Limited releases showed that vortex-entrained Sensor Fish usually experienced higher pressure fluctuations, a larger percentage of severe events, and much more rapid angular velocities. These results differ from those of the live fish study in which the injury rate was low for the fish released upstream of the vortex. The difference may result from the possibility that the fish were not entrained into the vortex because a previous study showed much higher injury rates for fish hand-tossed directly into the vortex from the deck.

Over the years, the USACE has collected a large amount of live fish, Sensor Fish, and CFD particletracking data during many spillway fish passage studies in the Columbia Basin. Another objective of this study was to organize these existing data into a single data repository and, through The Dalles Dam spillway case study, evaluate it as an efficient means for accessing and retrieving system-wide Sensor Fish and balloon-tagged live fish data for the USACE. The Dalles Dam spillway was selected as a case study to demonstrate the functionality of a comprehensive database containing Sensor Fish, live fish, and CFD simulation data because The Dalles Dam spillway is one of the most complex in the Pacific Northwest region and has the most comprehensive data set. The resulting database application is called the Fish Passage Analysis System (FishPAS). The database was implemented using Microsoft Access 
2003 and MathWorks MATLAB. It was configured to accommodate data from Sensor Fish, live fish, and CFD particle-tracking, as well as operations data for assessing fish passage conditions at turbines, spillways, and bypass facilities for all hydroelectric projects. The database interface provides researchers versatility in how the data can be queried and retrieved for different dam operation conditions. In September 2007, the USACE held a review meeting to evaluate the results of the case study. The recommendation from this meeting was that the database be extended for future studies in addition to existing balloon-tagged live fish data sets and the latest-generation 6-degree-of-freedom Sensor Fish data sets at other dams in the Columbia Basin. 


\section{Acknowledgments}

Funding for this project was provided by the U.S. Army Corps of Engineers, Portland District. The authors thank the following individuals for their contributions to this project:

- U.S. Army Corps of Engineers, Portland District

- Bob Wertheimer

- Laurie Ebner

- Mike Langeslay

- U.S. Army Corps of Engineers, Walla Walla District

- Martin Ahmann

- Marvin Shutters

- Ann Setter

- Normadeau Associates, Inc.

- Paul Heisy

- Joanne Fulmer

- Susan Haney

- Pacific Northwest National Laboratory

- Dennis Dauble

- Joanne Duncan

- Bob Mueller

- Andrea Currie

- Cindy Rakowski 



\section{Contents}

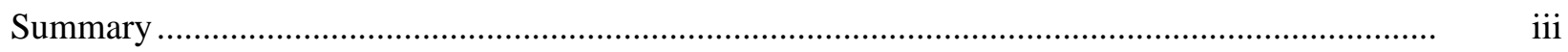

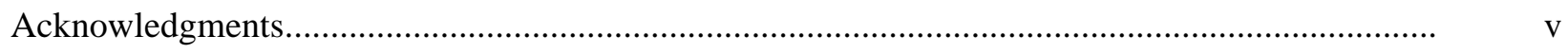

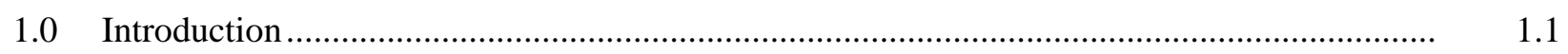

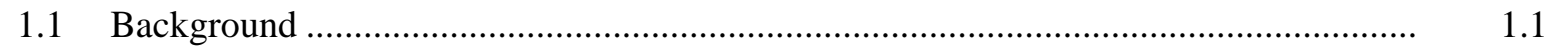

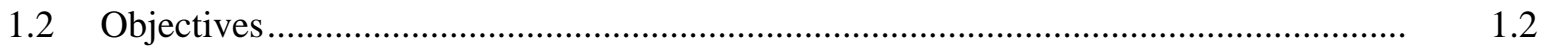

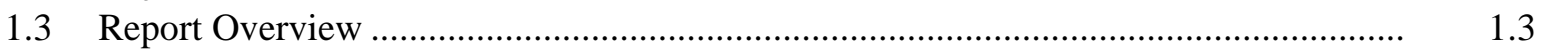

2.0 Characterization of Spillway Passage Conditions at The Dalles Dam in 2006..................... 2.1

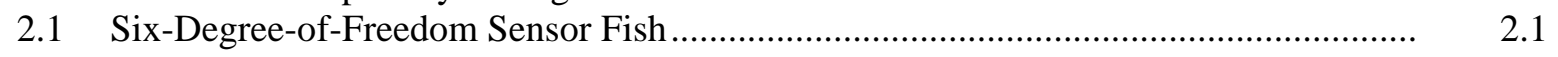

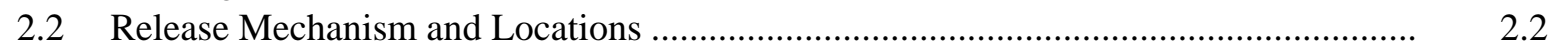

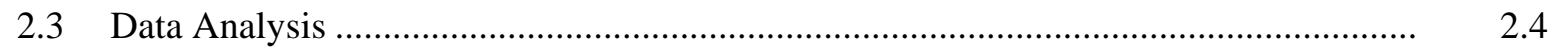

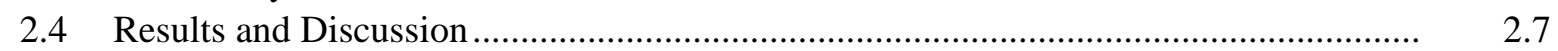

3.0 Fish Passage Analysis System (FishPAS): Requirements, Design, and Implementation ....... 3.1

3.1 Scope, Objectives and Risk Assessment ................................................................. 3.1

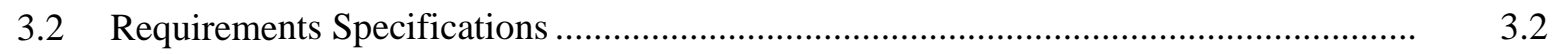

3.2.1 General Requirements .......................................................................... 3.2

3.2.2 Data Requirements .................................................................................... 3.2

3.2.3 Software Requirements ........................................................................... 3.3

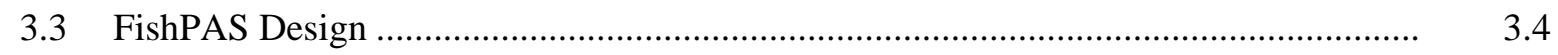

3.3.1 Data Repository ..................................................................................... $\quad 3.5$

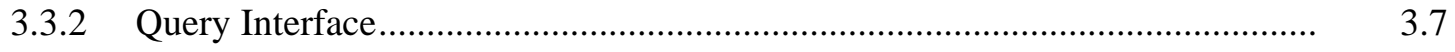

3.3.3 Analysis Tools.................................................................................. 3.8

3.3.4 Hardware and Software Integration ........................................................... 3.9

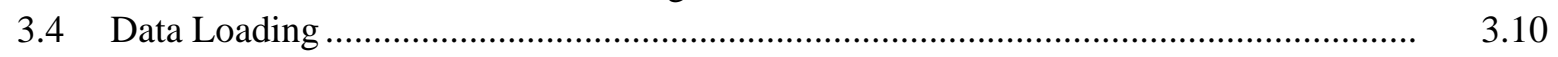

3.4.1 Loading Methods.................................................................................... 3.10

3.4.2 Lookup Lists.......................................................................................... $\quad 3.10$

3.4.3 Study, Treatments, and Locations …........................................................... 3.11

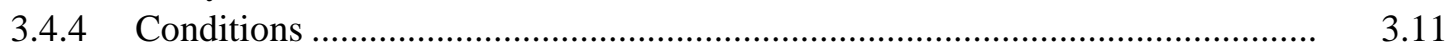

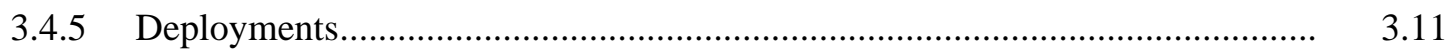

3.4.6 Time Histories ......................................................................................... 3.12

3.4.7 Attributes ........................................................................................ $\quad 3.12$

3.4.8 Miscellaneous Tables ........................................................................... $\quad 3.12$

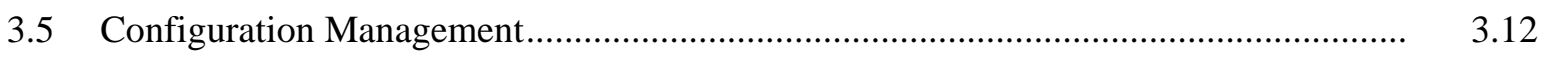

3.5.1 Roles and Responsibilities.......................................................................... 3.12

3.5.2 Data Validation and Verification .............................................................. 3.13

3.5.3 Baseline Configuration................................................................................ 3.13

3.5.4 Change Control ................................................................................ 3.13

3.5.5 Security .......................................................................................... $\quad 3.14$

3.6 Sample Queries Demonstrating Use of the Database................................................ 3.14

3.6.1 Application for Cross-Study Queries ........................................................... 3.15

3.6.2 Application for Operations Zone Evaluation ................................................ 3.16

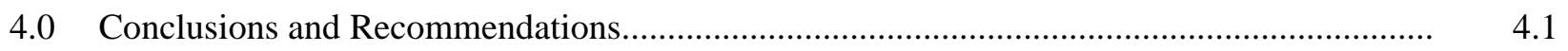




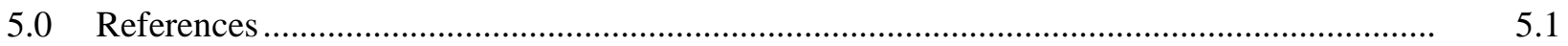

Appendix - Guidelines for Major and Minor Injury Classifications at Turb’N Tag Studies ............ A.1

\section{Figures}

2.1 The Sensor Fish Device Showing the Measurement Axes for the Three Rate Gyros, Three Linear Accelerometers, and Pressure and Temperature Transducers ..................................... 2.2

2.2 Cross Section of a Spillbay and Energy Dissipation Structures at The Dalles Dam................ 2.3

2.3 Top View of The Dalles Dam Spillway Showing the Locations of Bay 4, Bay 6, and the Vortex

2.4 Release Locations for the 12 Sensor Fish Released into the Surface of the Vortex

2.5 Comparison of Sensor Fish Measurements with Computational Fluid Dynamic Simulation Results During a Spillway Passage at Lower Monumental Dam Showing Pressure Time Histories and General Features of the Acceleration Magnitude Time Histories Similar to Those from CFD Simulation

2.6 Duration of Acceleration Within $70 \%$ of the Peak Value is Less Than 0.0075 Second for a Collision Event, Longer Than 0.0075 Second for a Shear Event.

2.7 Fitted Probability of Minor and Major Injuries As a Function of Sensor Fish Acceleration as Derived from Binary Logistic Regression.....

2.8 Example of Measurements by Sensor Fish Released from Mid-Bay at Bay 4

2.9 Example of Measurements by Sensor Fish Released from Mid-Bay at Bay 6

2.10 Example of Measurements by Sensor Fish Released from Upstream of the Vortex at Bay 6 Using Normandeau Associates Release Pipe

2.11 Example of Measurements by Sensor Fish into the Surface of the Vortex at Bay 6 Using the Surface Release Mechanism

2.12 Different Characteristic: Sensor Fish Released at Bay 4 Has Higher Pressure Peak at Transition Than Sensor Fish Released at Bay 6, Possibly Because Release Elevation at Bay 4 Is Lower Than That at Bay 6.

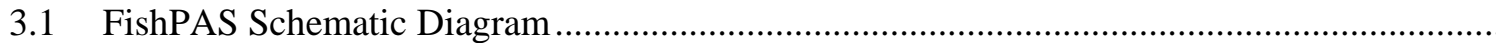

3.2 FishPAS Data Hierarchy

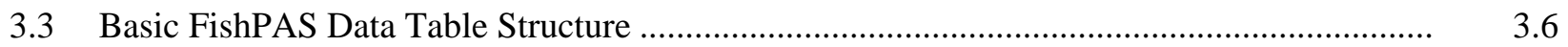

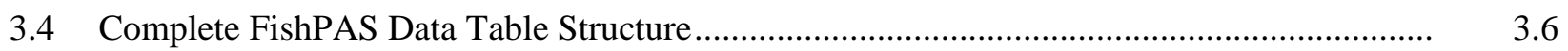

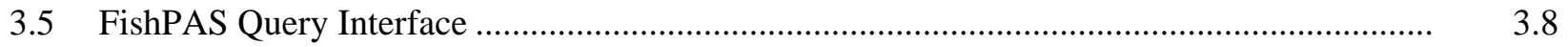

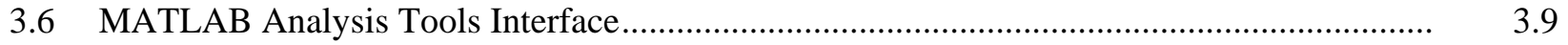

3.7 Condition Criteria Definition Interface .............................................................................. 3.15

3.8 FishPAS Reporting and Exporting Interface..................................................................... 3.16

3.9 Zone Definition for Fish Passage Condition Evaluation Using Discharge-Per-Bay and

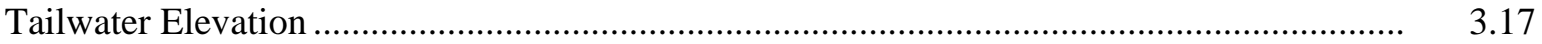

3.10 Injury Rate Comparison of Fish Released at Bay 4 Under Conditions of the Three Zones..... $\quad 3.17$ 


\section{Tables}

2.1 Summary of Hydraulic Conditions for the Four Sensor Fish Treatments................................ 2.4

2.2 Summary of Sensor Fish Measurements from Entry Points to Baffle Blocks ........................ 2.10

3.1 Lookup Tables in FishPAS .................................................................................................

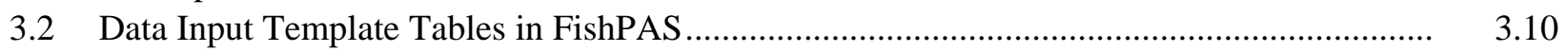

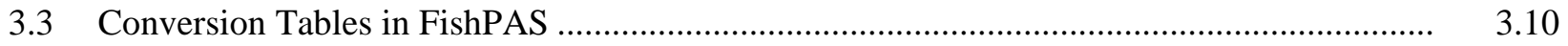

3.4 Data Input Template Tables in FishPAS ............................................................................... 3.14

3.5 Summary of Query Results for Middle Bay 4 Releases, with Release Elevation Less Than $133 \mathrm{ft}$ and Tailwater Elevation Less Than $80 \mathrm{ft}$................................................................ 



\subsection{Introduction}

The U.S. Army Corps of Engineers (USACE) has collected a large amount of data on live fish, Sensor Fish, and computational fluid dynamics (CFD) particle-tracking during many spillway fish passage studies in the Columbia Basin. For example, at The Dalles Dam spillway, nine balloon-tagged direct survival studies have been performed, with more than 12,000 live fish and 1,100 Sensor Fish released in those studies. These data were stored in separate formats and dispersed among several parties, including the USACE, the Pacific Northwest National Laboratory (PNNL), and Normandeau Associates, Inc., and have repeatedly proven difficult to consolidate for effective analysis. In 2004-2006, Richmond et al. (2007) developed a prototype database that was designed specifically to manage these disparate data by synthesizing them into a single database. The resulting prototype database demonstrated that this approach was an efficient means for accessing these data and developing tools for analyzing the data for The Dalles Dam spillway. The goal of the PNNL study described in this report was to complete a comprehensive database for The Dalles Dam spillway Sensor Fish and balloon-tagged live fish data sets, and through the evaluation and utilization of the case study database, determine if it is an efficient means for accessing and retrieving the system-wide data on Sensor Fish and live fish.

\subsection{Background}

The Sensor Fish is an autonomous measurement device developed at PNNL for the U.S. Department of Energy (DOE) and the USACE to better understand the physical conditions fish experience during passage through hydroelectric turbines and other dam passage routes. Since its initial development in 1997, the Sensor Fish has undergone several design changes to improve its function and extend the range of its use (Carlson and Duncan 2003).

The initial Sensor Fish device underwent major transformations in 2000 with polyvinyl chloride (PVC) tube housing and in 2002 with polycarbonate tube housing, providing an upgraded sensor package for deployment and rapid retrieval of data. These two generations of Sensor Fish devices were fieldtested at Bonneville, McNary, The Dalles, and Rock Island dams on the Columbia River. They were deployed in turbines, spillways, and sluiceways to measure changes in pressure and linear acceleration experienced by fish during dam passage.

A new design effort was completed in 2003. The resulting three-degree-of-freedom (3DOF) Sensor Fish reduced the cost of manufacture. It required less time for data downloading, consumed less power, recharged batteries more quickly, stored more data, and used nonproprietary software. It was deployed at The Dalles Dam spillway and Ice Harbor Dam spillway (Carlson and Duncan 2004) to characterize the fish passage conditions in 2003 and 2004.

The current model, the six-degree-of-freedom (6DOF) device, was successfully developed and deployed in February 2005 (Deng 2004, 2007). It measures the six components of motion: three components of linear acceleration (up-down, forward-back, and side-to-side) and three components of angular velocity (pitch, roll, and yaw). The Sensor Fish also measures absolute pressure and temperature. This Sensor Fish uses a sampling frequency of 2,000 Hz and a recording time of about 4 minutes (233 seconds). This compares to a sampling frequency of $200 \mathrm{~Hz}$ and a recording time of about 2 minutes with the previous models. This increased sampling frequency allows more accurate capture of the rapid change of motion and pressure that occurs, especially during collisions, and shear exposure, during passage through hydroelectric turbines and spill. It is $24.5 \mathrm{~mm}$ in diameter and $90 \mathrm{~mm}$ in length, weighs 
about $43 \mathrm{~g}$, roughly the same as a yearling salmon smolt, and is nearly neutrally buoyant in fresh water. This model has been deployed in spillways at The Dalles Dam (Deng et al. 2006) and at Ice Harbor and Lower Monumental dams (Carlson et al. 2006). It also has been field-tested for turbine passage at the John Day, Bonneville, Ice Harbor, Priest Rapids, and Wanapum dams.

Overall, to date, more than 30 Sensor Fish studies have been completed at 11 dams on the Columbia and Snake rivers: Bonneville, Grand Coulee, Ice Harbor, John Day, Lower Monumental, McNary, North Fork, Priest Rapids, Rock Island, The Dalles, and Wanapum. All these studies were completed with objectives specific to the dams at which the Sensor Fish devices were deployed. A synthesis of these system-wide data sets can significantly improve the accessibility of existing data, assist the design and data collection of future studies, and facilitate data processing. In addition, if integrated with direct survival and injury studies, the synthesis can assist in identifying and quantifying spillway environment conditions that provide safer passage for fish, thus establishing baseline conditions for comparison with spillway passage studies that may be conducted at other mainstem projects.

At the request of the USACE Portland District and Walla Walla District, PNNL convened a meeting on February 8, 2007, to discuss the need and scope of the synthesis effort. The Dalles Dam spillway was selected as a case study to demonstrate the functionality of a comprehensive database containing Sensor Fish, live fish, and CFD simulation data because The Dalles Dam spillway is one of the most complex in the Pacific Northwest region and has the most comprehensive data sets, for both Sensor Fish and live fish. The USACE requested that the database be designed for primary data use (the use for which the data were acquired) but not preclude secondary use (the use of data for researchers outside the primary organization and with objectives beyond those identified in the acquiring project's scope of work). In September 2007, another meeting was held to review the results of the case study, and the USACE participants recommended that the database be extended to system-wide use.

\subsection{Objectives}

The objectives of this study were to

- Characterize live test fish exposure conditions of The Dalles Dam spillway using Sensor Fish. Sensor Fish data before 2005 were acquired using the old generations of Sensor Fish and had been processed using metrics developed for those devices. Therefore, priority was given to the 2006 study in which Sensor Fish were released to evaluate the passage conditions in Bay 4 and Bay 6 at high discharges. The frequency of occurrence, location, and magnitude of severe exposure events such as collisions and shear were determined for different spill environment regions. Based on extensive laboratory studies conducted with funding from the DOE Wind \& Hydropower Technologies Program, we have developed a response relationship between shear injury (Deng et al. 2005) and Sensor Fish measurements for juvenile salmon subjected to different shear flow exposure mechanisms in a laboratory environment. The response relationship was applied to the 2006 deployments to estimate the trend for shear injuries during spillway passage and compare with live fish injuries. Note that a similar response relationship for collisions is not currently available.

- Synthesize The Dalles Dam spillway passage data including live fish, Sensor Fish, and CFD particletracking data with relevant hydraulic operation data for spillbays, turbines, and other water passage routes. Live fish data include both survival and injury, with injury classifications consistent with those used in the DOE laboratory investigations. Sensor Fish data consist of the primary time-history records from the Sensor Fish itself and secondary metadata describing Sensor Fish deployment details 
and dam operational conditions. Characteristic results such as timing and regions of severe events also are included as a separate attribute table. Only CFD particle-tracking data prior to 2005 are included in the database and analysis because CFD work to support Sensor Fish studies initially planned for 2006 were not funded.

- Organize these existing data in a single data repository and, through The Dalles Dam case study, evaluate it as an efficient means for accessing and retrieving system-wide data for the USACE. The database will be designed for primary users but will not preclude secondary users. It will be configured to handle live fish data in addition to Sensor Fish data.

- Using The Dalles Dam data sets as representative samples of data sets for other locations and passage routes, provide a mechanism and platform of study design, data acquisition and analysis, and data treatment for system-wide projects and future evaluation studies of fish passage conditions. Test and evaluate these database and data analysis features for system-wide application as The Dalles Dam case study is conducted.

\subsection{Report Overview}

Chapter 2 addresses the characterization of spillway passage conditions at The Dalles Dam in 2006 using Sensor Fish. Chapter 3 describes the requirements, design, and implementation of the Fish Passage Analysis System (FishPAS). Chapter 4 contains conclusions and recommendations. Chapter 5 provides a list of the references cited in this report. An Appendix contains guidelines for major and minor injury classifications at Turb'N Tag studies used for the database. 



\subsection{Characterization of Spillway Passage Conditions at The Dalles Dam in 2006}

The Dalles Dam is at river kilometer 306 on the mainstem Columbia River. The powerhouse was completed in 1957 and is sited on the border between the states of Oregon and Washington. The Dalles Dam consists of a 22-unit powerhouse, a 23-gate spillway, and a navigation lock. The configuration of this facility is such that the spillway is perpendicular to the river, while the powerhouse is parallel to the river. The spillway has an overall length of $1,370 \mathrm{ft}$ and contains 23 gates, each $50 \mathrm{ft}$ wide. Spill is regulated by tainter gates that pass water at a maximum depth of approximately $40 \mathrm{ft}$ below the upstream water surface. Water discharged through the tainter gates plunges approximately $50 \mathrm{ft}$ to the stilling basin. A single row of 9-ft-high by 10 -ft-wide concrete baffle blocks is located in the stilling basin to dissipate energy. An end sill lies downstream of the baffle blocks; this structure is a 13-ft-high vertical wall and lies approximately $10 \mathrm{ft}$ below normal tailwater elevation.

At The Dalles Dam, the survival of juvenile salmonids that pass through the spillway is lower than survival through many of the other spillways at other mainstem Columbia and Snake River dams. In addition, results from a direct survival and injury test showed relatively high direct survival and low injury for fish passing Bays 2 and 4 but lower than expected survival for fish passing through Bay 6 (Normandeau Associates and Skalski 2005). Some unique features are associated with Bay 6, including a large vortex that forms upstream on the south side of the spillbay approach near the south intake nose pier. Passage studies in 2004 indicated that approximately 25\% of the fish passing through the spillway passed through Bay 6 and that survival through that bay was lower than for fish passing through Bays 1 through 4. Furthermore, a three-dimensional acoustic tag study at The Dalles Dam indicated that a large majority of fish passing through Bay 6 passed on the south side of that bay. During winter 2004-2005, a hydraulic modeling effort at the USACE Engineer Research and Development Center (ERDC) showed that suspending a stop log in Bay 6 prevented formation of the large vortex. During February 2005, a brief test at The Dalles Dam was conducted, which verified the physical model results.

The original objective of this study was to characterize the exposure conditions live fish experience during passage through Bays 4 and 6 with and without the presence of a vortex suppression device in Bay 6. Releases were originally planned for three locations: mid-Bay 4, mid-Bay 6, and the southern edge of Bay 6. However, because of higher than expected river discharge and failure of the submerged release pipe at the southern edge of Bay 6, the scope was changed to deployment at only two release locations without the vortex suppression device: mid-Bay 4 and mid-Bay 6. A limited number of Sensor Fish were also released to the upstream of the vortex and into the surface of the vortex after the mid-bay releases were completed.

\subsection{Six-Degree-of-Freedom Sensor Fish}

The Sensor Fish (Figure 2.1) is an autonomous device being developed at PNNL for DOE and USACE to better understand the physical conditions fish experience during passage through hydropower turbines and other dam bypass alternatives (Carlson and Duncan 2003; Deng et al. 2004). It is $24.5 \mathrm{~mm}$ in diameter and $90 \mathrm{~mm}$ in length and weighs $42 \mathrm{~g}$. It is roughly the same size as a yearling 


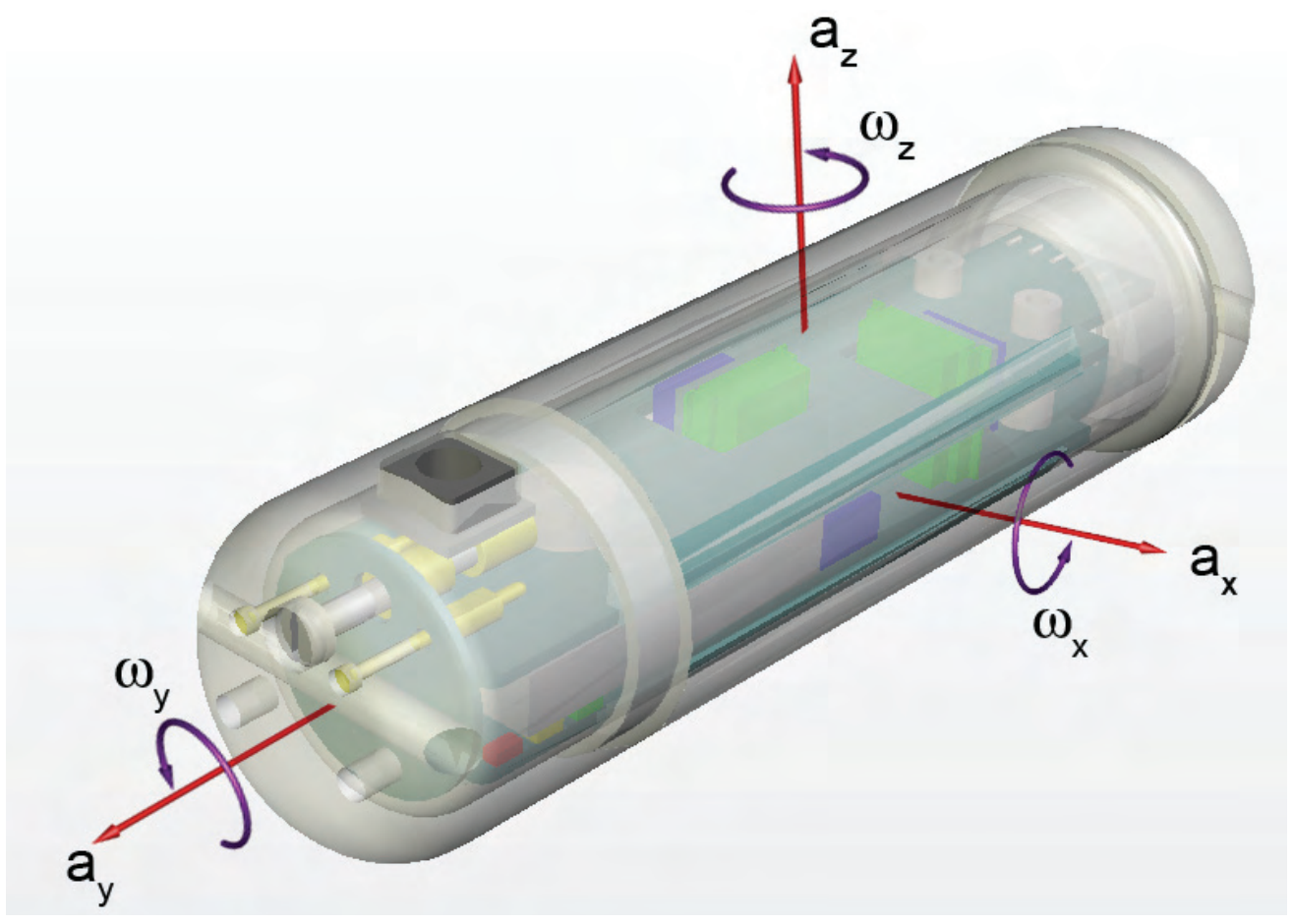

Figure 2.1. The Sensor Fish Device Showing the Measurement Axes for the Three Rate Gyros (that measure angular velocity, $\Omega$ ), Three Linear Accelerometers (that measure the acceleration, a), and Pressure and Temperature Transducers

salmon smolt and, like a fish, is nearly neutrally buoyant in fresh water. It measures the three components of linear acceleration (up-down, forward-back, and side-to-side), the three components of angular velocity (pitch, roll, and yaw), and the absolute pressure and temperature at a sampling frequency of 2,000 Hz (Deng et al. 2004, 2007). All devices were tested in a calibration apparatus. The accuracy of the pressure sensor was determined to be within $0.2 \mathrm{psi}$, and the relative errors of both the linear acceleration and angular velocity measurements were determined to be less than $5 \%$ based on laboratory acceptance tests.

\subsection{Release Mechanism and Locations}

In April 2006, 25 and 31 Sensor Fish Devices were released concurrently with balloon-tagged live fish in Bays 4 and 6 at a target injection elevation of $131 \mathrm{ft}$ (10 ft above ogee) using a 4-in.-diameter flexible hose threaded inside a 6-in.-diameter steel pipe (Figure 2.2; Normandeau Associates and Skalski 2006). Six Sensor Fish were also deployed upstream of the vortex in Bay 6 by a 3-in.-diameter flexible hose mounted to the nose pier (Figure 2.3). The terminus of the hose was approximately $1 \mathrm{ft}$ off the pier and $1 \mathrm{ft}$ above the water surface. In addition, with a surface-deployment mechanism, 12 Sensor Fish were released in the surface at the upstream edge, left edge, downstream edge, and the core of the vortex at Bay 6 (Figure 2.4; Deng et al. 2006). The spill volume for the mid-bay releases at Bays 4 and 6 ranged from 16.2 to $19.9 \mathrm{kcfs}$ at the test bay, the total spill volume was from 100 to $140 \mathrm{kcfs}$, and total project discharge was from 269.7 to $385.4 \mathrm{kcfs}$ (Table 2.1). The spill volume ranged from 16.3 to $16.5 \mathrm{kcfs}$, and total project discharge ranged 324 to $342 \mathrm{kcfs}$ for the releases upstream of the vortex. The total spill was 110 to $124 \mathrm{kcfs}$, and the spill volume at Bay 6 was 18 and $20 \mathrm{kcfs}$ for the surface-entrained Sensor Fish releases. 


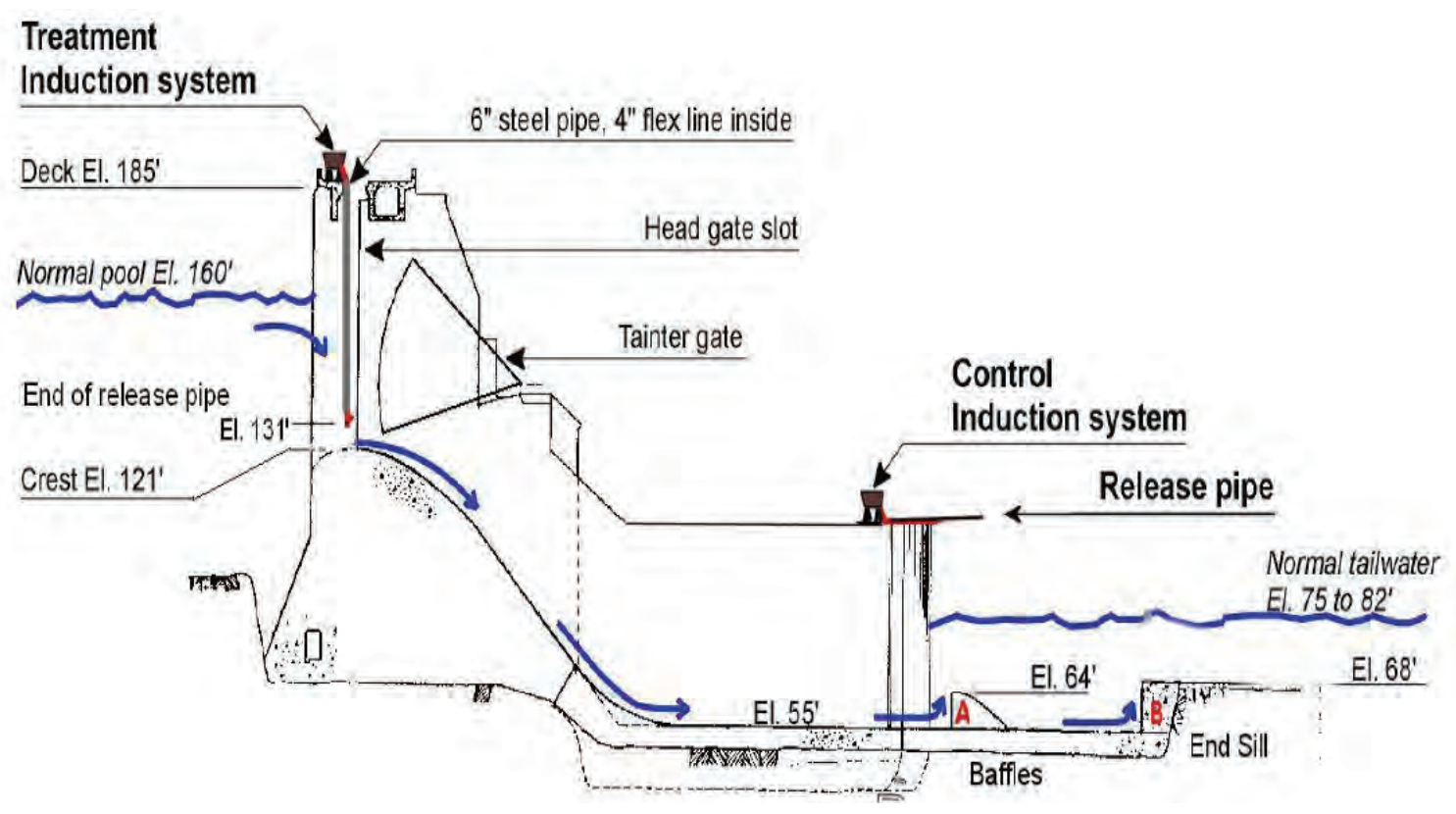

Figure 2.2. Cross Section of a Spillbay and Energy Dissipation Structures at The Dalles Dam (adapted from Normadeau Associates and Skalski 2006)

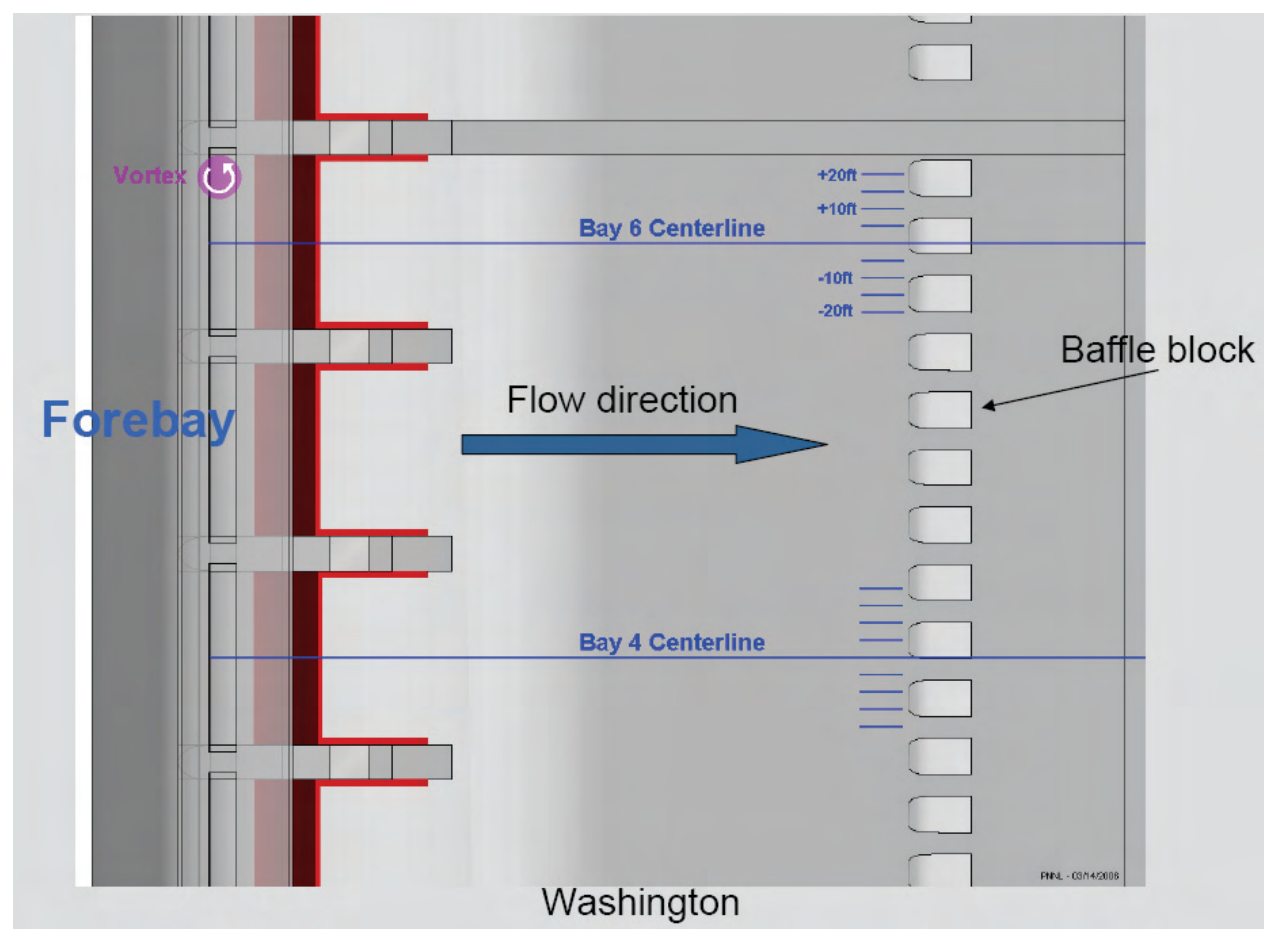

Figure 2.3. Top View of The Dalles Dam Spillway Showing the Locations of Bay 4, Bay 6, and the Vortex 


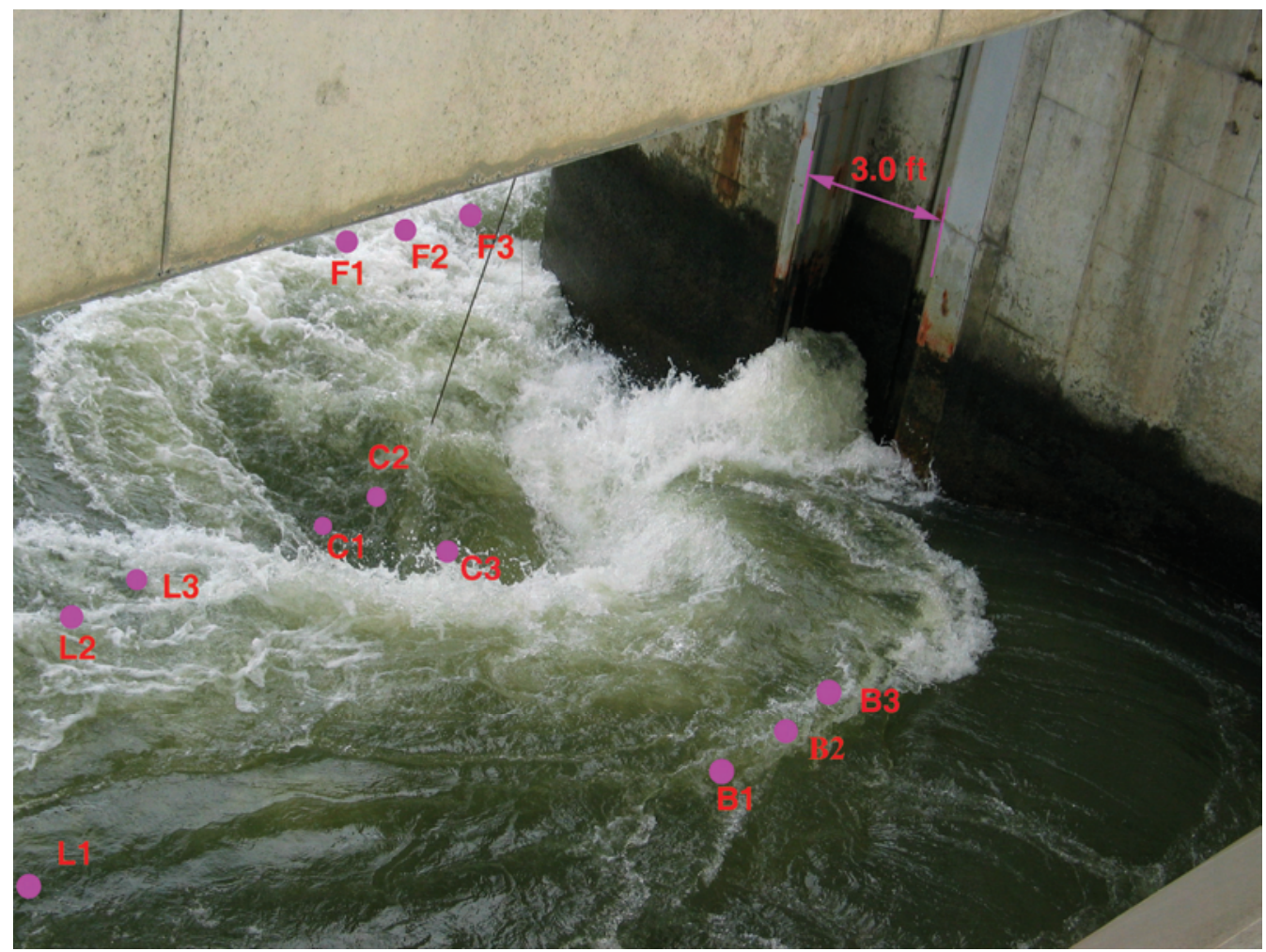

Figure 2.4. Release Locations for the 12 Sensor Fish Released into the Surface of the Vortex

Table 2.1. Summary of Hydraulic Conditions for the Four Sensor Fish Treatments

\begin{tabular}{|c|c|c|c|c|c|c|c|c|}
\hline Bay & Position & $\begin{array}{c}\text { Target } \\
\text { Release } \\
\text { Elevation (ft) }\end{array}$ & $\begin{array}{c}\text { Number of } \\
\text { Samples }\end{array}$ & $\begin{array}{l}\text { Test Bay } \\
\text { Spill (kcfs) }\end{array}$ & $\begin{array}{c}\text { Total } \\
\text { Spill } \\
\text { (kcfs) }\end{array}$ & $\begin{array}{c}\text { Total } \\
\text { Project } \\
\text { (kcfs) }\end{array}$ & $\begin{array}{c}\text { Forebay } \\
\text { Elevation }(\mathrm{ft})\end{array}$ & $\begin{array}{l}\text { Tailwater } \\
\text { Elevation }(\mathrm{ft})\end{array}$ \\
\hline Bay 4 & Mid-bay & 131 & 25 & $16.6-19.9$ & $100-140$ & $270-383$ & $157.6-159.6$ & $81.4-85.7$ \\
\hline Bay 6 & $\begin{array}{l}\text { Mid-bay } \\
\text { Upstream }\end{array}$ & 131 & 31 & 16.2-19.9 & $107-140$ & 271-385 & 157.3-158.9 & 81.7-85.7 \\
\hline Bay 6 & of vortex & Near surface & 6 & $16.3-16.5$ & 101-111 & 324-342 & $157.9-158.9$ & $84.0-84.7$ \\
\hline Bay 6 & Vortex & Surface & 12 & 18.3-19.8 & $110-124$ & 272-314 & 157.9-158.4 & 81.7-83.2 \\
\hline
\end{tabular}

\subsection{Data Analysis}

Sensor Fish data sets consist of time histories of pressure, acceleration ( $x, y$, and $z$ axes), angular motion (pitch, roll, and yaw), temperature, time, and battery status extending from the time of release through the period of data acquisition programmed prior to release of the Sensor Fish. Pressure samples are used to estimate the depth of the Sensor Fish (when hydrostatic pressure conditions can be assumed) and identify characteristic signatures to divide into segments corresponding to specific locations (zones) that extend from Sensor Fish injection to the end of the specific passage. For a typical Sensor Fish released in the middle of a spillbay, readily identifiable are the time of passage from the injection pipe exit into the forebay, passage under the tainter gate, time in the spillway chute, transition to the tailrace, and passage in the tailrace (Figure 2.5). These distinctive signature events are used to estimate the probable location and time of collision or shear exposure events and timing information for integration with CFD results. 


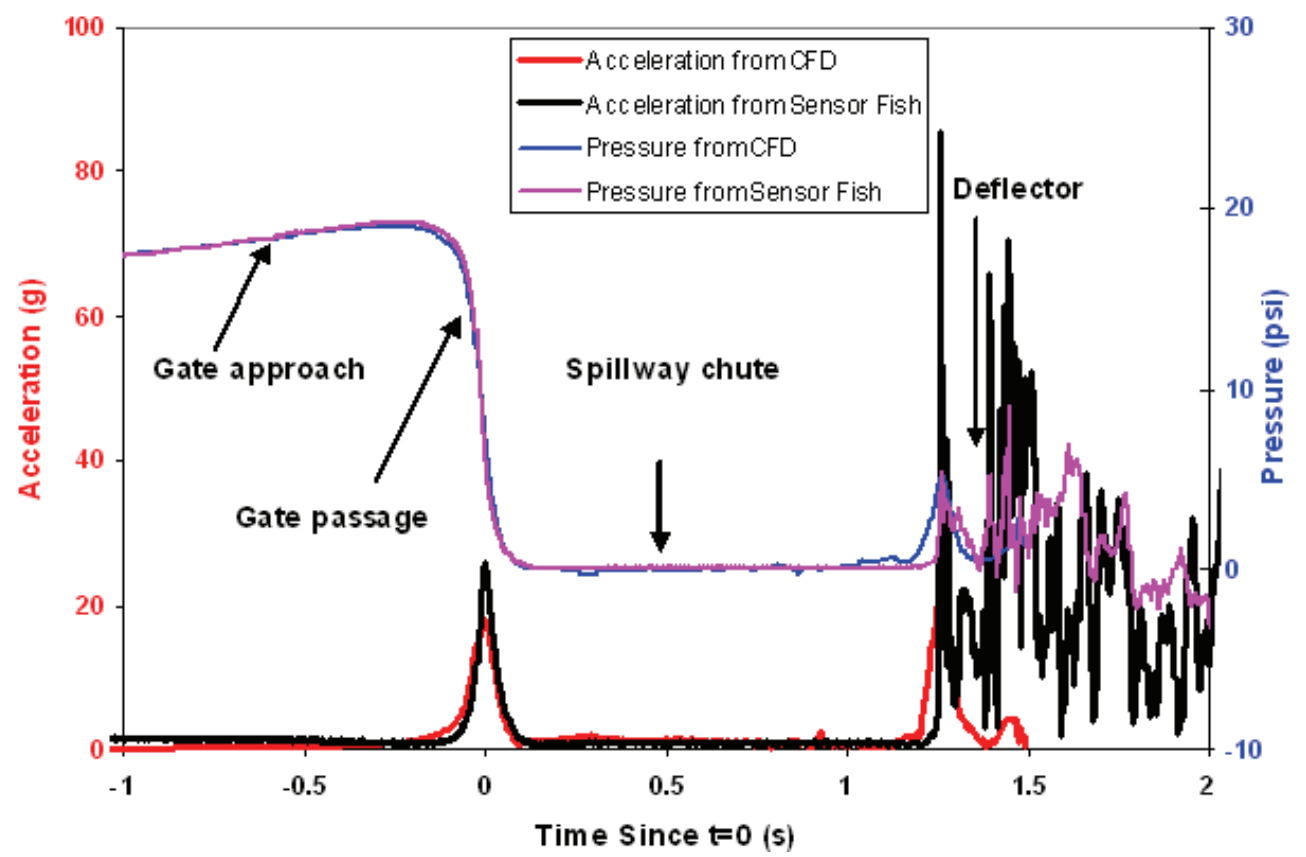

Figure 2.5. Comparison of Sensor Fish Measurements with Computational Fluid Dynamic (CFD) Simulation Results During a Spillway Passage at Lower Monumental Dam Showing Pressure Time Histories and General Features of the Acceleration Magnitude Time Histories Similar to Those from CFD Simulation

When Sensor Fish are in contact with solid structures or impacted by turbulent shear, high-amplitude impulses occur in the acceleration and rotational velocity time history. If the acceleration reaches a certain threshold, the exposure is counted as a severe exposure event. Based on some previous laboratory and field studies (Deng et al 2005; Carlson et al 2006), an acceleration threshold of $95 \mathrm{~g}$ was selected as the definition of severe events. The identification of an event as collision or shear is based on the different characteristics of acceleration and rotational velocities during the significant exposure event. Observations of Sensor Fish response in a laboratory flume show that a collision event creates a much narrower peak in acceleration and rotational velocity than does a shear event. In this study, peak duration is defined as the duration of acceleration within $70 \%$ of the peak value, and collision and shear events are distinguished by the following criteria: 1) the significant event is a collision event when peak duration is less than 0.0075 second (Figure 2.6(a)); 2) the significant event is a shear event when peak duration is longer than 0.0075 second (Figure 2.6(b)). Pressure and rotational measurements are then used for validation of the classification (Figure 2.6(c) and (d)).

In a set of separate laboratory studies (Deng et al 2005; Dauble et al 2006), juvenile fall Chinook salmon (Oncorhynchus tshawythscha) and Sensor Fish were exposed to turbulent shear flows to establish correlation metrics between Sensor Fish measurements and live fish injuries under two exposure scenarios. In the fast-fish-to-slow-water scenario, test fish were carried by the fast-moving water of a submerged turbulent jet into the standing water of a flume. In the slow-fish-to-fast-water scenario, test fish were introduced into a turbulent jet from standing water through an introduction tube placed just 

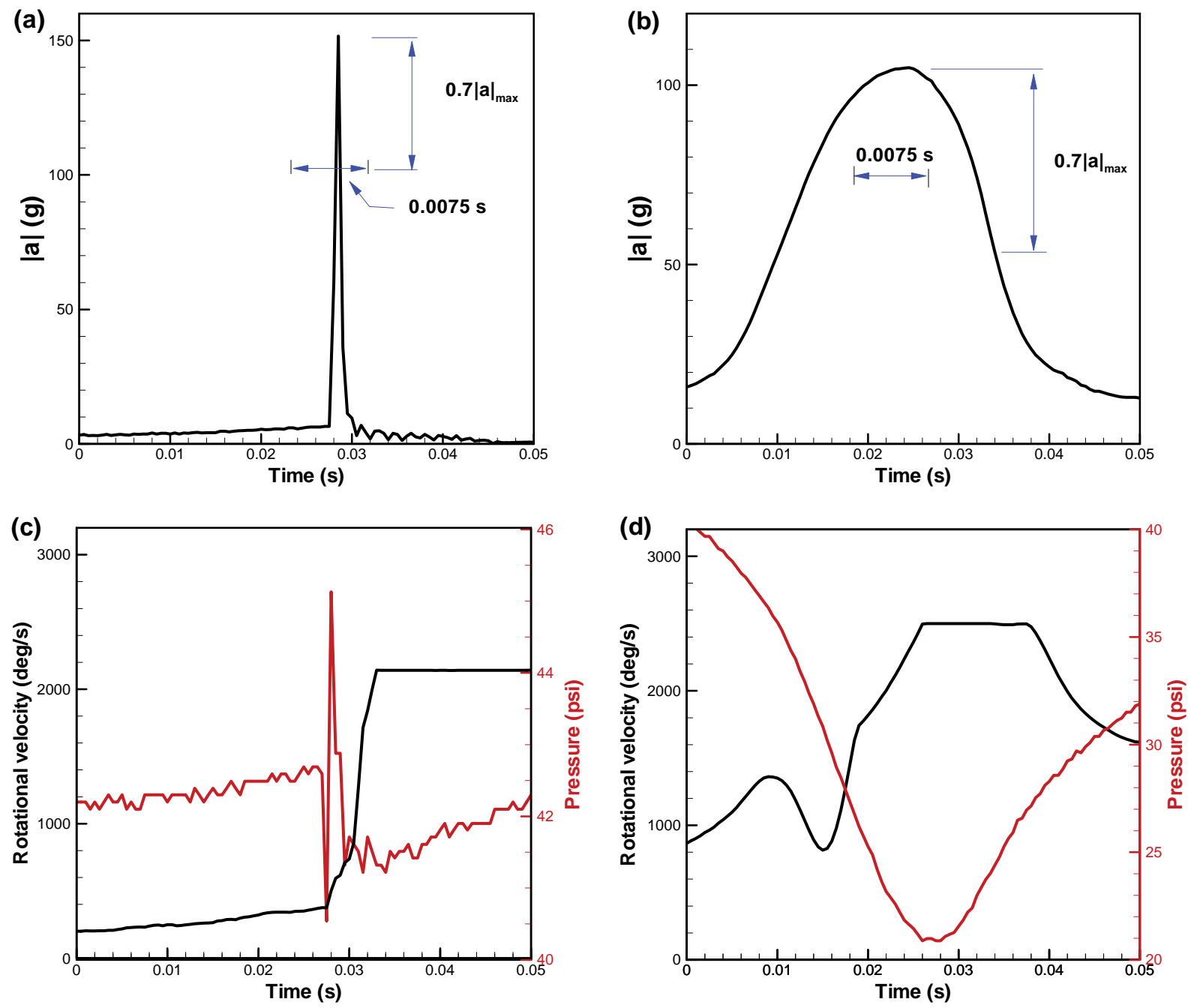

Figure 2.6. Duration of Acceleration Within 70\% of the Peak Value is (a) Less Than 0.0075 Second for a Collision Event, (b) Longer Than 0.0075 Second for a Shear Event. Pressure and rotation increase more dramatically during (c) a collision event than during (d) a shear event.

outside the edge of the jet. At the present time, it is not possible to determine from Sensor Fish data which type of exposure scenario is represented by a given shear event. However, in a spillway passage, the Sensor Fish is entrained predominantly in fast-moving water; thus it is likely that most shear exposures would be of the fast-fish-to-slow-water type. Figure 2.7 shows the probability of minor and major biological injury for Sensor Fish acceleration measurements for the fast-fish-to-slow-water exposure.

Standard deviations of the probabilities of collisions or shear events were derived using bootstrapping. Bootstrapping is a statistical method for estimating a sampling distribution by creating more bootstrapped samples by drawing, with replacement, from the original sample (Efron and Tibshirani 1993). This method is very useful in the evaluation of standard errors and confidence intervals of parameters such as mean, median, proportion, odds ratio, correlation, or regression coefficient, especially where there are no other simple alternatives. 


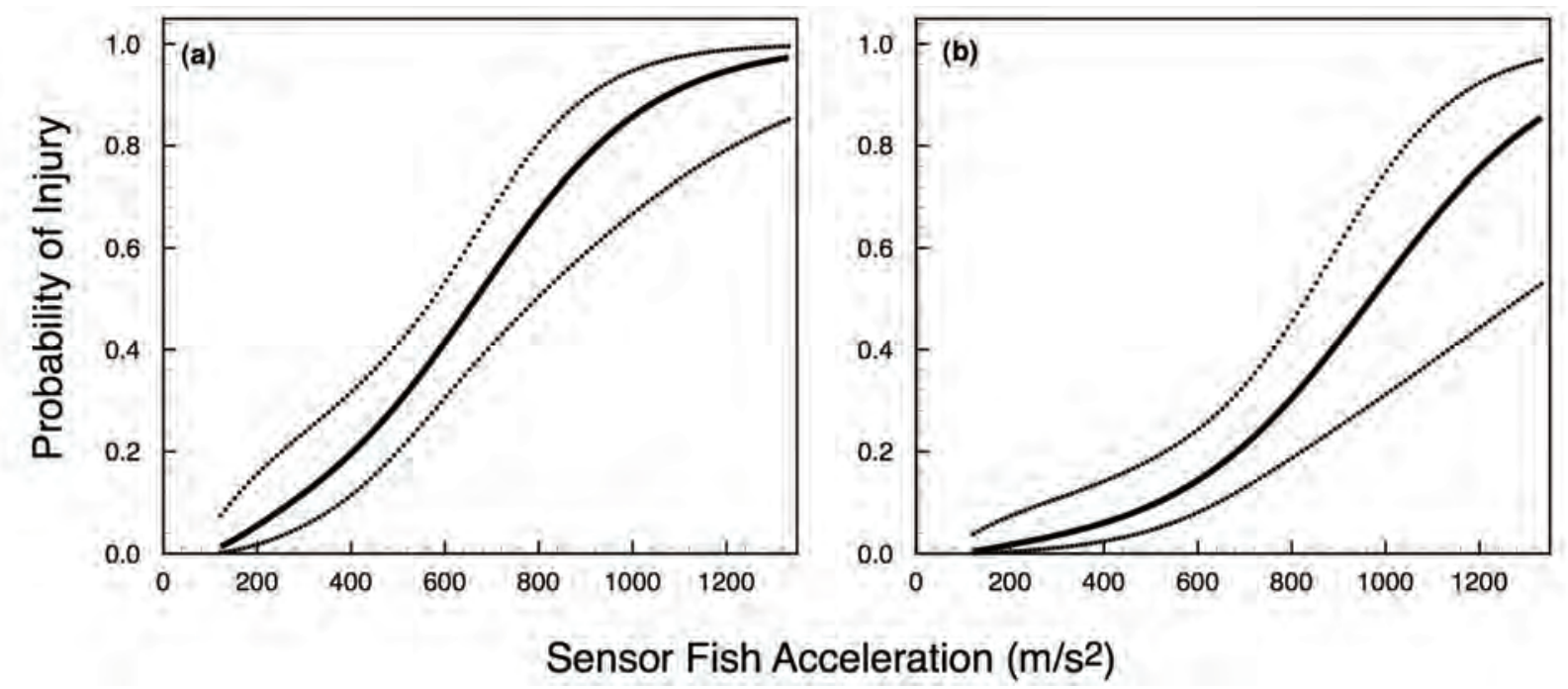

Figure 2.7. Fitted Probability of Minor and Major Injuries As a Function of Sensor Fish Acceleration (with 95\% predictive confidence intervals) as Derived from Binary Logistic Regression.

(a) Minor injury for fast-fish-to-slow-water scenario; (b) major injury for fast-fish-to-slowwater scenario.

\subsection{Results and Discussion}

For typical releases, only a few seconds were required for a Sensor Fish to travel from the entry point to the baffle block in the stilling basin. Some distinctive features characterized entry into the forebay, passage under the tainter gate, time in the spillway chute, transition to the tailrace, and approach to the baffle blocks (Figures 2.8 through 2.11). There was an increase of acceleration and angular velocity when the Sensor Fish passed under the tainter gate. For typical mid-bay releases without significant exposure events, the passage was smooth, with very small acceleration and angular velocity in the spillbay chute until it entered the tailwater and was close to the baffle blocks (Figures 2.8 and 2.9). In the tailrace immediately after the spillway, both the acceleration and angular velocity increased significantly because of the turbulent nature in this flow region. In comparison with measurements of Sensor Fish released at mid-bay, vortex-passage Sensor Fish usually experienced higher pressure fluctuations and much more rapid angular velocities (Figures 2.10 and 2.11).

Release elevations for the mid-bay releases were estimated assuming hydrostatic conditions. The accuracy of these estimates can be evaluated by more detailed flow information (for example, from CFD) in the forebay (Carlson et al. 2006). For the high-volume spills in this study, the release elevation error assuming hydrostatic conditions at The Dalles Dam can be in the range of 2 to $3 \mathrm{ft}$ (Richmond et al. 2007). Assuming hydrostatic conditions, the estimated release elevations computed using sensor fish pressures for Bay 4 and Bay 6 were $135 \mathrm{ft}$ and $138 \mathrm{ft}$, respectively. Using results from a two-dimensional CFD simulation of The Dalles tainter gate indicates that the hydrostatic estimate may be in error by approximately $3 \mathrm{ft}$ for Bay 4 at $18 \mathrm{kcfs}$ spill. In other words, the sensor fish may have been released at the elevations closer to the target elevation at $131 \mathrm{ft}$ (10 ft above the ogee) than what we initially estimated. 

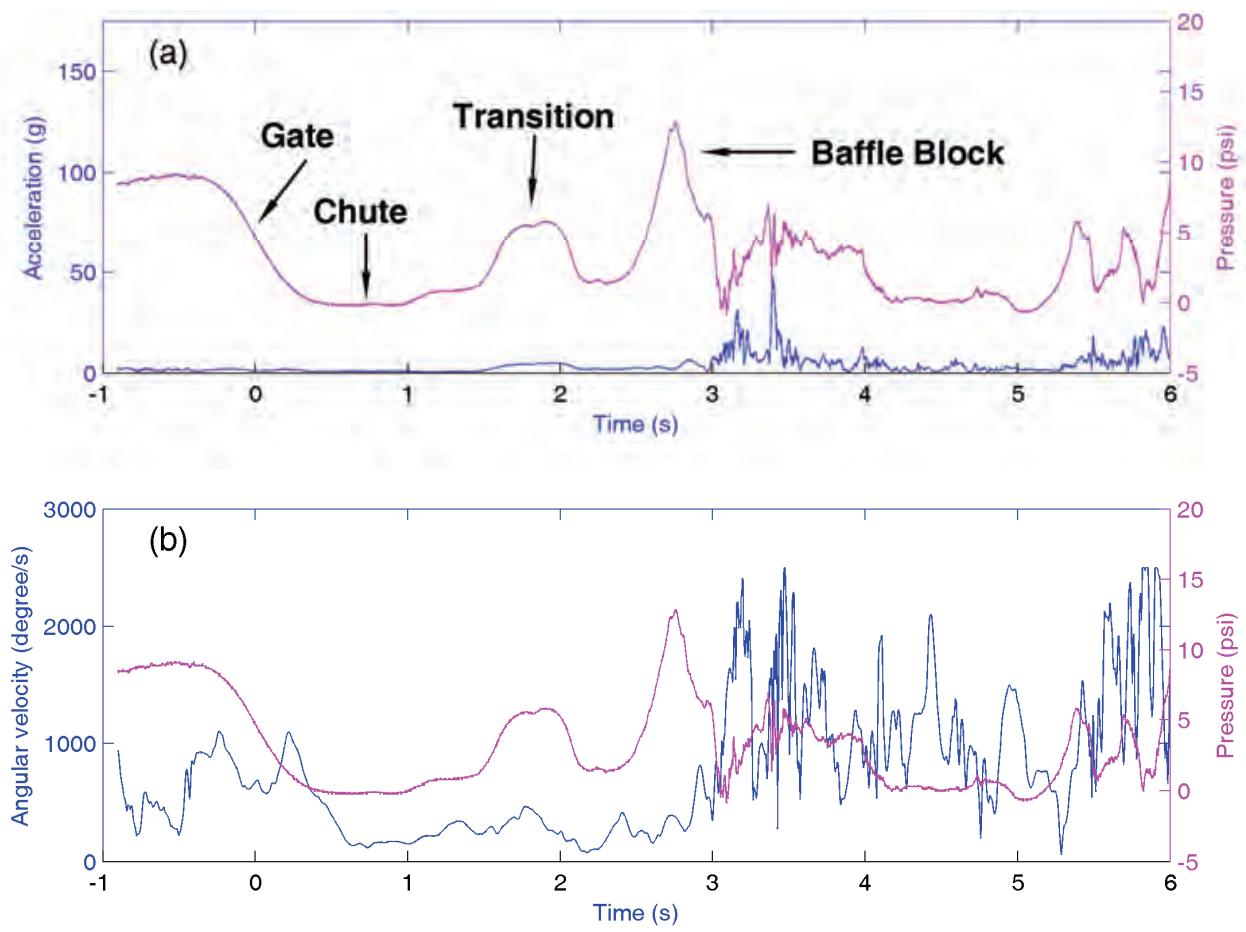

Figure 2.8. Example of Measurements by Sensor Fish Released from Mid-Bay at Bay 4
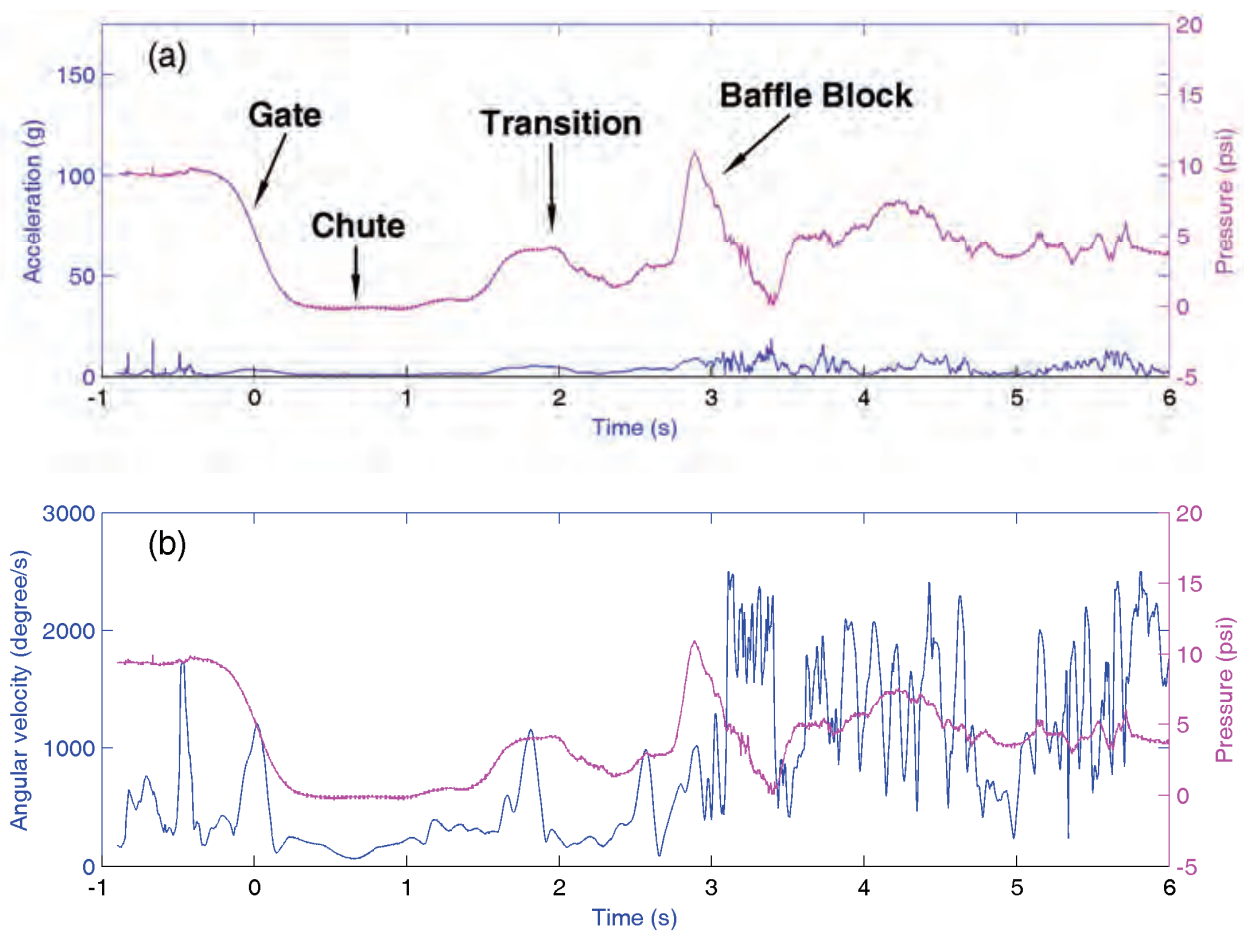

Figure 2.9. Example of Measurements by Sensor Fish Released from Mid-Bay at Bay 6 

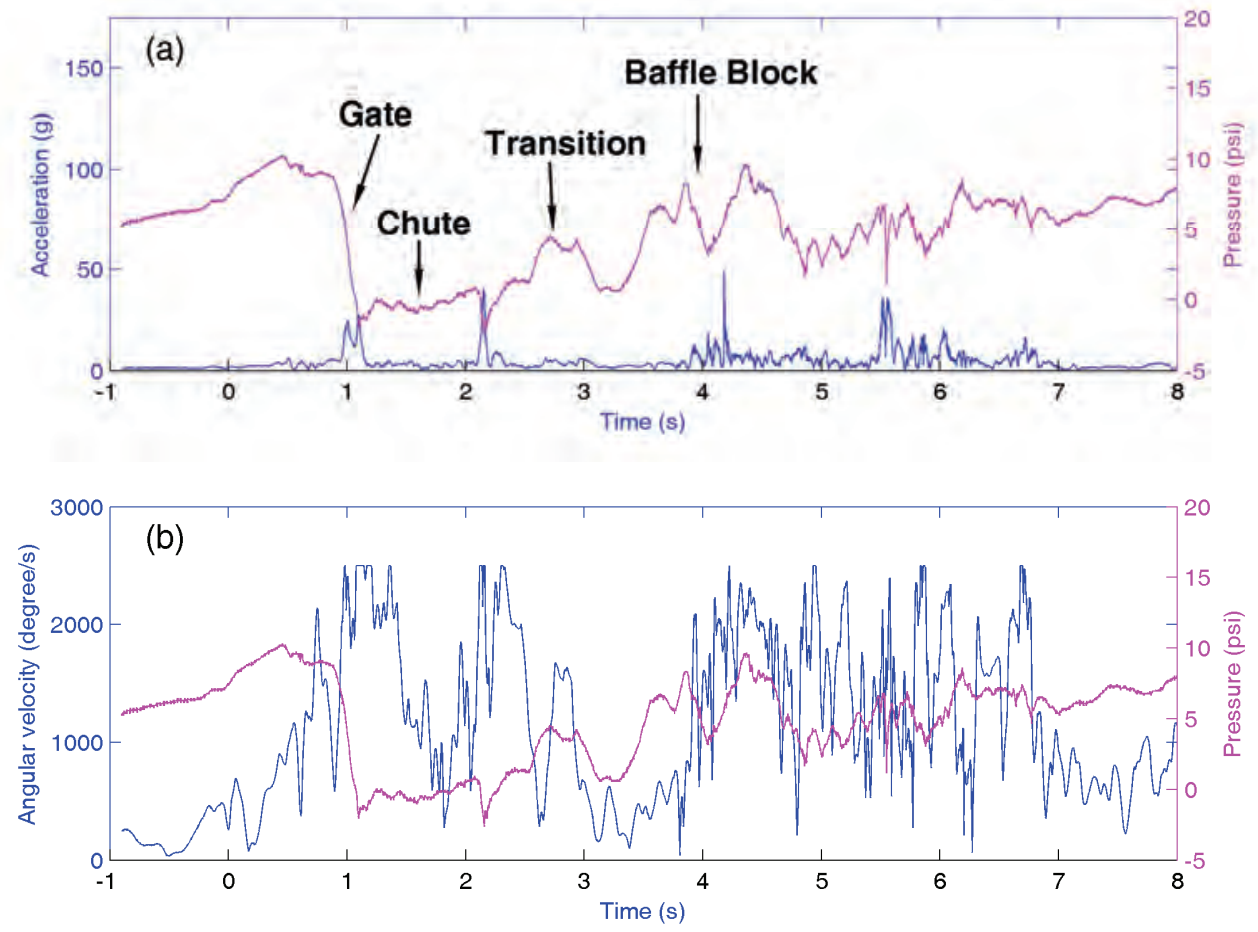

Figure 2.10. Example of Measurements by Sensor Fish Released from Upstream of the Vortex at Bay 6 Using Normandeau Associates Release Pipe
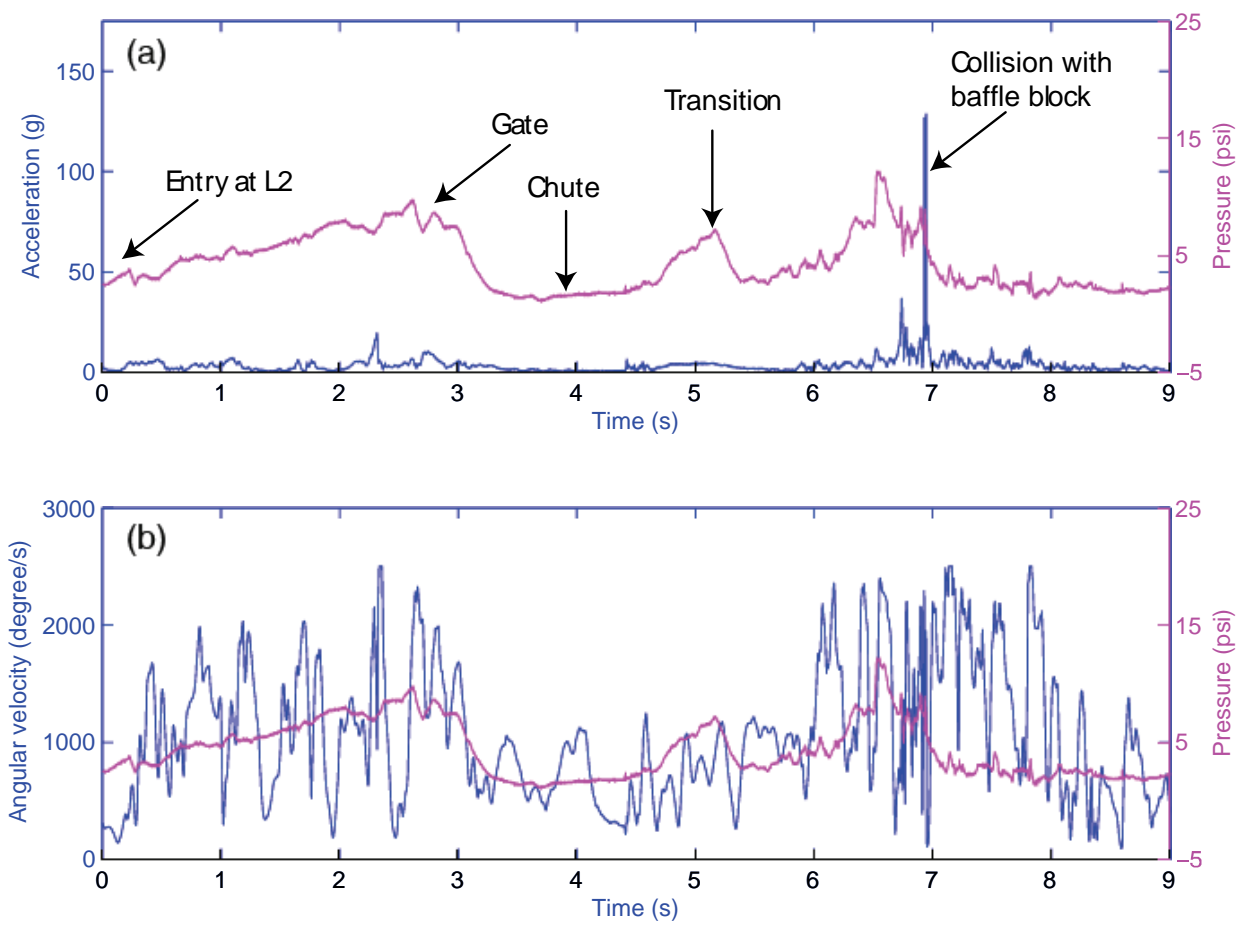

Figure 2.11. Example of Measurements by Sensor Fish into the Surface of the Vortex at Bay 6 Using the Surface Release Mechanism 
Sensor Fish released at mid-Bay 4 experienced a higher pressure spike at transition than Sensor Fish released at Bay 6 (Figure 2.12). It could be caused by a difference in release elevations between these two bays. An alternative explanation is that the Bay 6 vortex causes a local reduction in static pressure, which would lead to an apparent elevation change between the two release bays. If a Sensor Fish were released deeper at a lower elevation, it could approach the transition region in the lower water column where a higher pressure zone usually exists. At this time, we have no conclusive evidence from which to determine whether the release pipe exits were actually placed at the target elevations or whether the apparent elevation differences are caused by the non-hydrostatic pressure fields that exist at Bays 4 and 6 .

An acceleration value of $95 \mathrm{~g}$ was selected as the threshold for severe events. During passage from the entry points to the baffle blocks, $40 \%$ of Sensor Fish released into mid-Bay 4 experienced at least one severe event, while 16\% of Sensor Fish released into mid-Bay 6 had at least one severe event (Table 2.2). Therefore, Sensor Fish released from Bay 6 had smoother passage than those released from Bay 4, which is consistent with the findings of the live fish study in which fish released from Bay 4 sustained higher injury rates than fish from Bay 6 and the survival rate at Bay 6 was higher than that at Bay 4 (Normandeau Associates and Skalski 2006). For the releases related to the vortex, it is worthwhile to point out that the sample sizes were very small because it was a pilot study. The limited releases showed that vortex-entrained Sensor Fish usually experienced a larger percentage of severe events compared to the Sensor Fish released mid-bay. It is different from the findings of the live fish study that the injury rate was low for the fish released upstream of the vortex. The difference may result from the possibility that the fish have not been entrained into the vortex because a previous study showed much higher injury rates for fish hand-tossed directly into the vortex (Normandeau Associates and Skalski 2005).

The response curves in Figure 2.7 were used to estimate the rates of shear injury from the time histories of Sensor Fish acceleration measurements in the middle bay releases. The vortex releases were not evaluated due to small sample sizes. The overall predicted major shear injury rates were $12.1 \%$ for Bay 4 and 5.1\% for Bay 6. This compares to observations by Normandeau Associates and Skalski (2006) of $2.8 \%$ and $0.8 \%$ for Bays 4 and 6 , respectively. Estimated shear injury rates using the Sensor Fish measurements were overpredicted because the response curves in Figure 2.7 were developed for worstcase scenarios (i.e., maximum impact). A similar set of response relationships for collision injury is not currently available.

Table 2.2. Summary of Sensor Fish Measurements from Entry Points to Baffle Blocks

\begin{tabular}{|c|c|c|c|c|c|c|c|}
\hline Bay & Release Location & $\begin{array}{l}\text { Number } \\
\text { of } \\
\text { Releases }\end{array}$ & $\begin{array}{c}\text { Total Severe } \\
\text { Events } \\
(|\mathrm{a}|>95 \mathrm{~g})\end{array}$ & $\begin{array}{c}\text { Severe } \\
\text { Collision }\end{array}$ & Severe Shear & $\begin{array}{l}\text { Live } \\
\text { Fish } \\
\text { Injury } \\
\text { Rate }\end{array}$ & $\begin{array}{c}\text { 48-hr } \\
\text { Survival }\end{array}$ \\
\hline 4 & Mid-bay & 25 & $40 \% \pm 9 \%$ & $24 \% \pm 8 \%$ & $16 \% \pm 7 \%$ & $4 \%$ & $98.50 \%$ \\
\hline 6 & Mid-bay & 31 & $16 \% \pm 7 \%$ & $13 \% \pm 6 \%$ & $3 \% \pm 3 \%$ & $1 \%$ & $99.00 \%$ \\
\hline 6 & Upstream of vortex & 6 & $50 \% \pm 20 \%$ & $33 \% \pm 19 \%$ & $17 \% \pm 15 \%$ & $2 \%$ & $98.60 \%$ \\
\hline 6 & Vortex & 12 & $66 \% \pm 14 \%$ & $50 \% \pm 15 \%$ & $16 \% \pm 11 \%$ & N/A & N/A \\
\hline
\end{tabular}




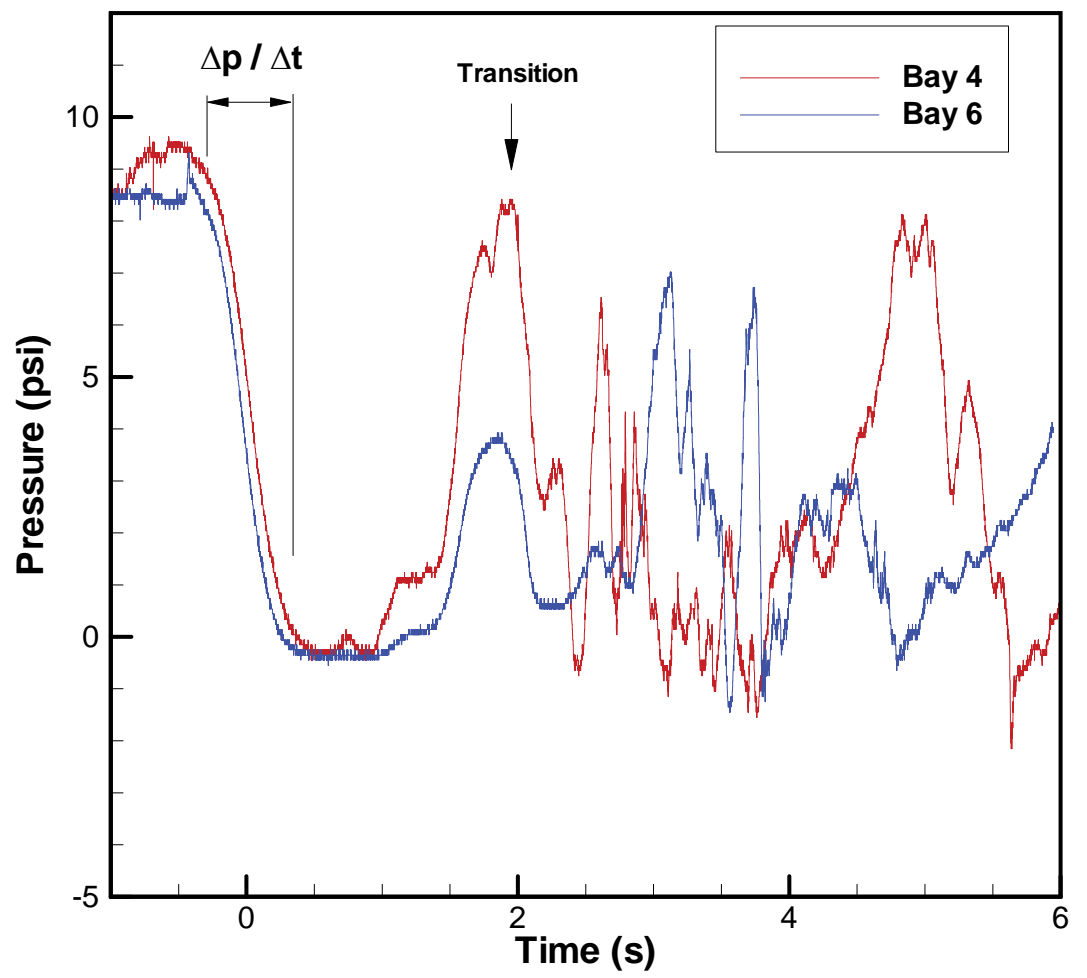

Figure 2.12. Different Characteristic: Sensor Fish Released at Bay 4 Has Higher Pressure Peak at Transition Than Sensor Fish Released at Bay 6, Possibly Because Release Elevation at Bay 4 Is Lower Than That at Bay 6. 



\subsection{Fish Passage Analysis System (FishPAS): Requirements, Design, and Implementation}

One of the main goals of this study was to complete a comprehensive database for The Dalles Dam spillway Sensor Fish and balloon-tagged live fish data sets and, through The Dalles Dam spillway case study, evaluate the database as an efficient means for accessing and retrieving system-wide data. The name for this database application is Fish Passage Analysis System (FishPAS). This section is the primary reference for the FishPAS application and includes the requirements, design, and implementation associated with the development and operation of the software system.

\subsection{Scope, Objectives and Risk Assessment}

FishPAS includes three types of data collected as part of fish passage studies conducted at hydroelectric dams-live-fish passage data, Sensor Fish logger data, and CFD particle-tracking data. Live-fish data consist of observations of the biological impact to juvenile salmonids as a consequence of passing through a particular route of interest (e.g., spillway, turbine). These data are collected to measure, in terms of injuries and mortalities, the direct physical harm caused by the passage. In the past, Normandeau Associates, Inc. (e.g., Normandeau Associates and Skalski 2005) has collected these data for the USACE.

Sensor Fish data sets consist of time histories of pressure, acceleration ( $x, y$, and $z$ axes), angular motion (pitch, roll, and yaw), temperature, time, and battery status, extending from the time of release through the period of data acquisition programmed prior to release of the Sensor Fish. PNNL developed and deploys the Sensor Fish, usually concurrently with live fish in collaboration with Normandeau Associates, Inc.

Particle tracking software generates simulated paths through passage routes of interest from CFD models (Richmond et al. 2007). These particles move under the influence of the model flow field and collect information about the flow environment along their path. The particles, in effect, can simulate the data obtained with Sensor Fish with the added benefits that, unlike the Sensor Fish, the particle location is always known and the number and location of deployments is almost unlimited. PNNL developed the particle-tracking algorithms and "deploys" the particles in output from CFD models.

These data sets are useful individually, but each has its disadvantages; they can provide more insights when they are analyzed together. This is practical only if the data can be efficiently retrieved together in a logical and effective manner. Therefore, the general goals for the FishPAS project are to

- Organize existing data on live fish, Sensor Fish, and CFD particle-tracking into a single, secure database.

- Provide an efficient means for accessing the data by researchers.

- Develop tools for analyzing the data.

- Ensure data integrity through a quality assurance process.

In accordance with PNNL software development procedures (the Software subject area of the PNNL Standards-Based Management System), application development must follow the Institute of Electrical 
and Electronics Engineers (IEEE) standard for developing a life-cycle process for project software (IEEE 2006), with the level of detail for each step graded according to the risk associated with the product. Risk criteria include potential for creating detrimental safety and health conditions, degrading the environment, compromising information security, and exceeding budget due to uncertainty of requirements or technology.

Analyses using FishPAS are anticipated to be limited to fundamental research. Course-of-action decisions that affect the environment may be based in part on results from FishPAS, but these decisions are subject to independent review on many levels. Therefore, the risk of FishPAS creating negative safety or environmental consequences is small.

Risk associated with information security, primarily the loss of irreproducible data, is of concern, as the data stored in FishPAS represents a significant financial investment. This risk is addressed through configuration management as described in Section 3.5.

\subsection{Requirements Specifications}

System requirements were developed through interaction with researchers associated with passage study data. The numbers and types of features implemented in this initial version of FishPAS were limited not only by budget constraints but also by the philosophy that real-world experience with a basic system is the best method of refining detailed requirements. Therefore, this current specification describes a foundation upon which subsequent refinements will be made as user experience develops.

\subsubsection{General Requirements}

The following general requirements apply to this database project. The application should

- Operate on a Microsoft Windows-based personal computer using software that is readily available and familiar to researchers at PNNL and USACE.

- Be accessible to at least three users simultaneously through the PNNL local area network (LAN).

- Provide a retrieval time of less than 10 seconds for individual data sets.

- Have an initial data storage capacity of 10 gigabytes (GB), with expected growth of 10 GB per year.

- Have reasonable safeguards to prevent inadvertent data loss or modification.

\subsubsection{Data Requirements}

The database must have structures for storing the raw forms of data for live fish, Sensor Fish, and CFD particle-tracking, additional information necessary to link and use these data sets (metadata), and certain results of data analysis. The following types of data sets must be accommodated:

- Acquired data:

- live-fish data - These data consist of injury and survival information for live-fish releases. The injuries shall be classified in a way that individual maladies (e.g., eye or operculum injuries) can be resolved. In addition, PNNL and Normandeau Associates collaborated to develop new guidelines for injury classification for better comparison with the laboratory studies conducted by PNNL. The details of the guideline are listed in the Appendix. Fish survival at several time 
intervals after recapture shall be recorded. Metadata must include descriptive information such as species, lot, length, and age. A photograph of the fish, if available, may be stored.

- Sensor Fish data - Sensor Fish records consist of time histories of pressure and motion data collected by the logger during a deployment. The fields of interest are sample time, pressure, triaxial acceleration, and triaxial rotational velocity (the last available only with the current six-degree-of-freedom unit). Provisions for incorporating new fields that could be available in future generations of Sensor Fish should be made. Metadata must include descriptive information such as model and ID number of each Sensor Fish.

- particle-tracking data - Particle-tracking data consist of time histories of various hydraulic parameters, typically including flow velocity, particle velocity, and pressure, obtained at each time step along a particle path computed through a CFD model. Metadata must include information about the CFD model and tracking code, such as software version, boundary conditions, and turbulence model.

- Metadata:

- dam operations - Operations data, such as total river flow, forebay and tailrace elevations, and individual unit discharges, must be associated with all data sets. The data structure for storage of this information must be flexible to accommodate an unknown number of operational parameters.

- test conditions - Information necessary to identify test conditions and allow comparisons with data from similar test conditions, such as release location (pipe elevation, spillbay, offset) and time, recovery information (time, location), and treatment block, must be captured.

- calibration and validation - Each Sensor Fish deployment and particle-tracking data set must be traceable to a calibration or model validation record, which would reference a calibration/validation document.

- Derived data:

- attributes - Information generated during the analysis of Sensor Fish and particle-tracking data must be stored as attributes associated with one or more deployments. An attribute is a value that characterizes some aspect of a deployment (e.g., collision count metric, location of pressure nadir). A single deployment may have many attributes, and the data structure for storage of this information must be flexible to accommodate an unknown number of possible attributes. All attributes must be traceable to their originator.

\subsubsection{Software Requirements}

The application must have tools to perform the following tasks:

- Prepare and load all the types of data present in the database.

- Retrieve any stored data set into a table for viewing on the computer screen.

- Query data by one or more criteria (e.g., deployment date, treatment, dam operations, injury type) based on any fields in the database except for time history fields.

- Plot one or more Sensor Fish data sets on the screen, allowing manipulation of the axis scales, time-scale offsets of each data set, and point probing of the plot.

- Assign user attributes to a Sensor Fish deployment using the plot screen. 
- Allow users to create custom treatment groups to pool deployments together efficiently.

- Export selected data to ASCII file format.

\subsection{FishPAS Design}

At the highest level, FishPAS may be subdivided into three major components: a Data Repository, a Query Interface, and Analysis Tools (Figure 3.1). In the typical work process, data are retrieved from the Data Repository using the Query Interface and passed on to the Analytical Tools for evaluation. Results of analyses may then be passed back to the Data Repository. The Query Interface is implemented in Microsoft Access, and the Analysis Tools component is accessed in MATLAB. The Data Repository includes data in both Access and MATLAB formats (products, respectively, of the Microsoft Corporation, Redmond, Washington, and The MathWorks, Inc., Natick, Massachusetts). These software package choices were driven by the experience of the developers and the analytical tools currently used by the researchers. In addition, the analysis tools in MATLAB were compiled into libraries. These libraries are then linked directly to the database without the need to run MATLAB, so no license for MATLAB is needed for the full functionalities of the database.

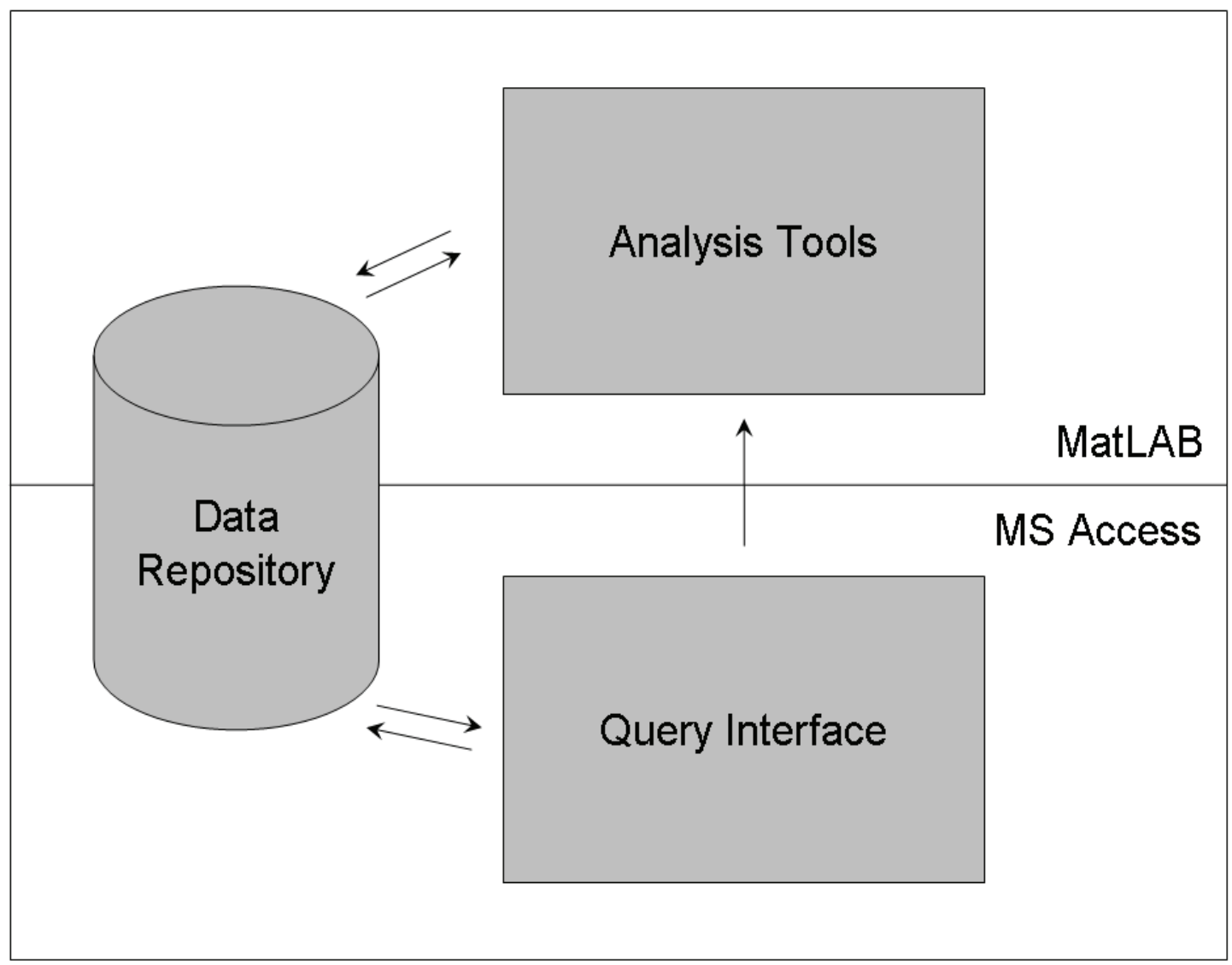

Figure 3.1. FishPAS Schematic Diagram 


\subsubsection{Data Repository}

The FishPAS Data Repository consists of Access data tables and MATLAB binary data files stored on a Microsoft Windows-based microcomputer. Data are organized into the general hierarchy shown in Figure 3.2. In Figure 3.2, each box represents a subject area and corresponds to one or more tables or files, as shown in a more explicit table structure diagram (Figure 3.3). Data in the Time History subject area, including Sensor Fish data-logger time histories and particle-tracking data, are stored in MATLAB binary data files rather than in Access, due to their large size and the requirements for rapid access. All other data are stored in data tables within a single Access .mdb file.

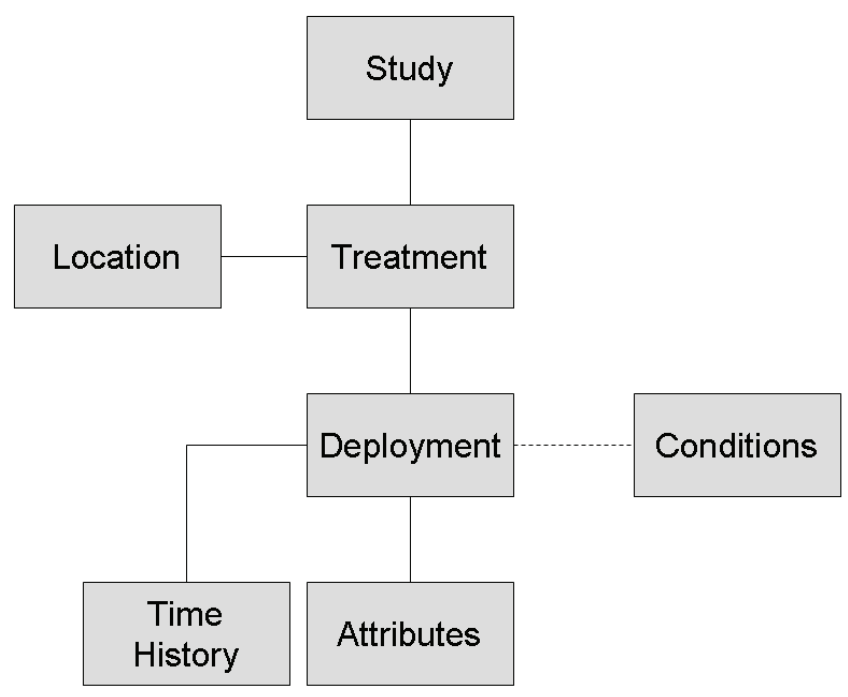

Figure 3.2. FishPAS Data Hierarchy

FishPAS also has numerous support tables called lookup lists. Lookup lists are tables that contain allowable values of a single key used in other database tables. Their purpose is to standardize field values to reduce the possibility of multiple values representing a single entity (e.g., this prevents the unit "ft" from being stored as "feet”, "Feet”, "FT”). To distinguish lookup lists from other tables, their names end in "List" (see Table 3.1 for a list of lookup tables). These tables have a referentially enforced one-to-many relationship with the tables they support (Figure 3.4).

The remainder of this section briefly describes each of the subject areas shown in Figure 3.2:

- Study - A study is a data collection effort conducted at a single hydroelectric project, usually under a single contract. All data in FishPAS belong to one, and only one, study. Information stored in this subject area includes the name and purpose of the study, the start and Table 3.1. Lookup Tables in FishPAS end dates, and the point of contact.

\begin{tabular}{c}
\hline Table Name \\
\hline tblAttributeList \\
tblCoordList \\
tblMalCauseList \\
tblMalCodeList \\
tblMalDesigList \\
tblMeasList \\
tblPersonList \\
tblProjectList \\
tblSFLoggerList \\
tblSpeciesList \\
tblSurvivalList \\
tblUnitsList
\end{tabular}




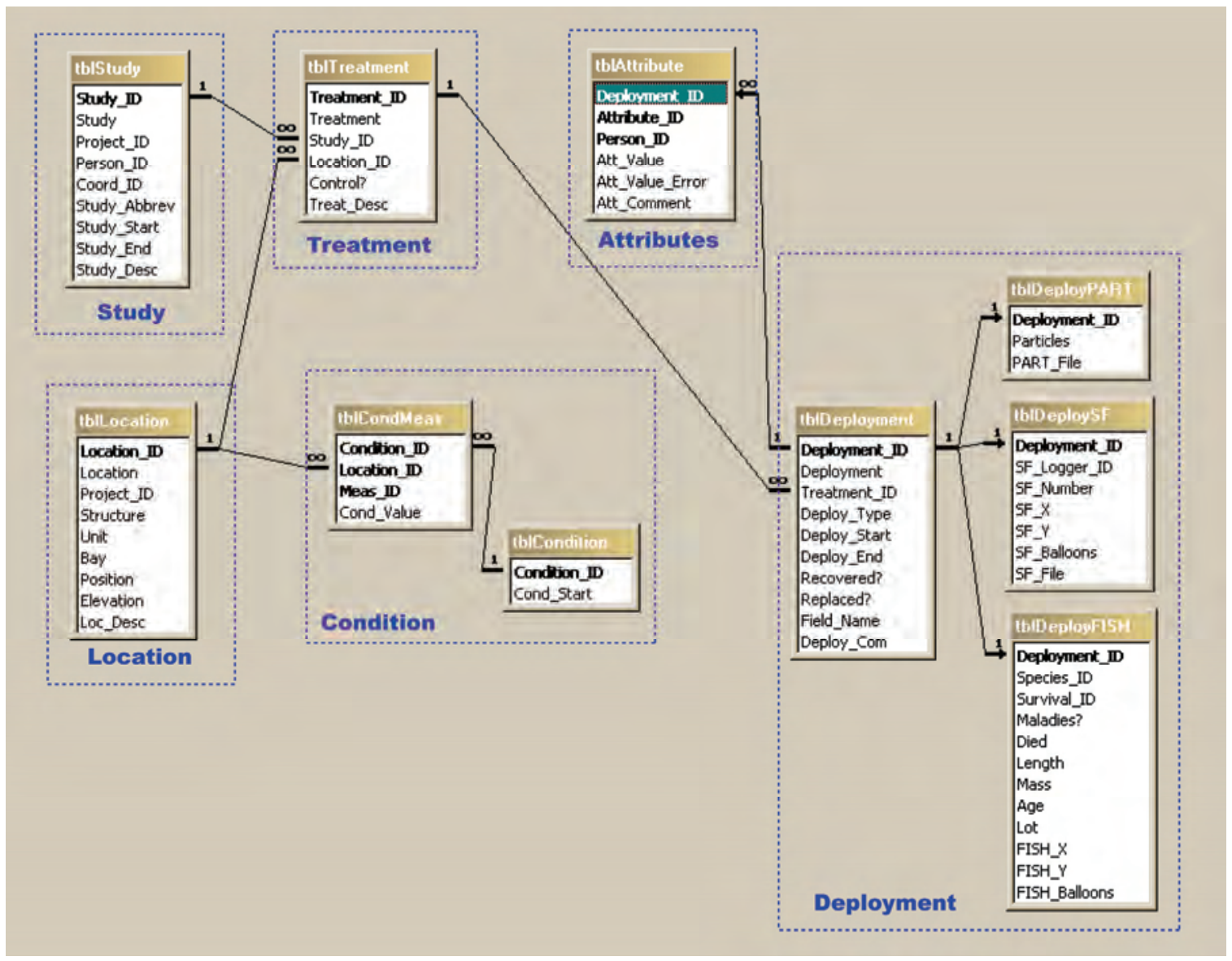

Figure 3.3. Basic FishPAS Data Table Structure

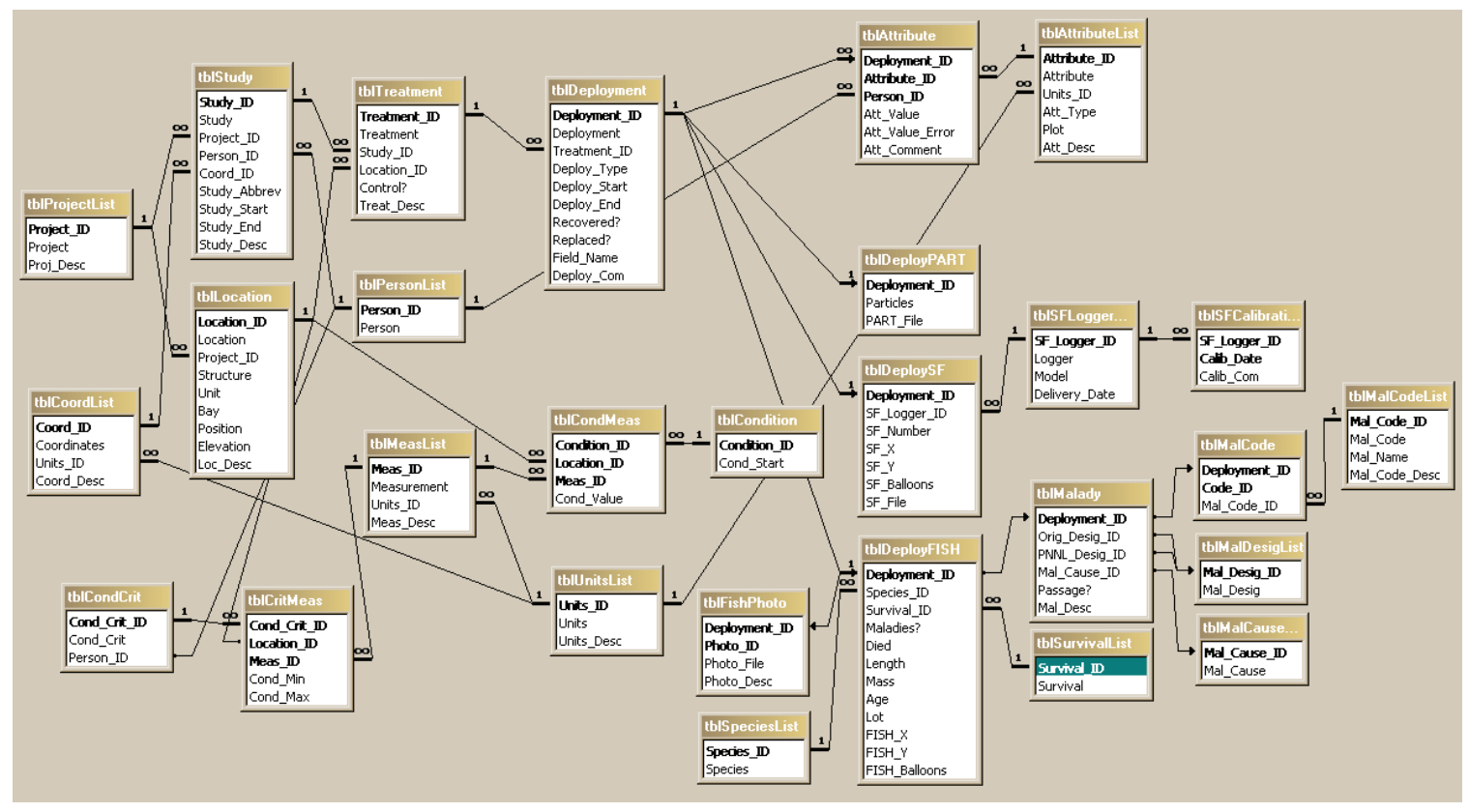

Figure 3.4. Complete FishPAS Data Table Structure 
- Treatment - A treatment is an experimental scenario being tested in the study. The treatment defines the conditions under which a group of data is collected. Conditions include deployment location, project operations, and, in the case of CFD particles, information about the model solution used. Although it is generally desirable to hold all conditions constant for a particular treatment, in practice this is rarely feasible. Therefore, the conditions subject area is associated with each deployment, through the deployment date, rather than with each treatment.

- Location - Each treatment is associated with a single deployment location, defined using a hierarchical specification. At the top of the hierarchy is the hydroelectric project (e.g., TDA). Subsequent levels include structure (e.g., powerhouse), unit (e.g., turbine unit 1), bay (Bay A), position (e.g., bay center, $10 \mathrm{ft}$ upstream of weir), and elevation above sea level (e.g., $130 \mathrm{ft}$ ).

- Deployment - A deployment is the release of a single live fish or Sensor Fish, or the release of a group of CFD particles from a single location. The information captured for each of these deployment types varies, but every deployment is associated with a single treatment and condition and has a release and recovery date/time. Deployment data specific to live fish include descriptions of the fish before release and injuries observed after recovery. Live fish may also have recovery coordinates and a reference to a photograph. Data specific to Sensor Fish include logger unit number, calibration information, recovery coordinates, and a reference to the MATLAB binary file containing the time history data. Data specific to CFD particle data include the number of particles in the release and a reference to the MATLAB binary file containing the time history data.

- Time History - Each time history data file contains the results from one Sensor Fish or CFD particle deployment. The data must include a time field. For Sensor Fish, other fields include triaxial acceleration, triaxial rotational velocity, and pressure. For CFD particles, fields include particle velocity, fluid velocity, pressure, and kinetic energy.

- Attributes - Users may assign attributes to any deployment. An attribute may be a calculated metric (e.g., collision count), the location of a significant event in a time history (e.g., pressure nadir), or just a marker for use in a query. The types of attributes are virtually unlimited because the attribute name is stored as a value, not as a field, in the database. Every attribute is associated with the user who assigned it.

- Conditions - This subject area defines the environmental conditions under which a deployment occurred. Relevant conditions include river stage elevations, powerhouse and spillway flow distributions, and possibly other measured characteristics specific to the study (e.g., turbine blade angle, CFD model characteristics). Because the need for and availability of experimental conditions varies from study to study, this subject area is flexible enough to accommodate a virtually unlimited number of condition measurements. This is implemented by storing the measurement types as values, rather than fields, in the database, thereby allowing the addition of new measurement types. Conditions are linked to deployments by date and time. Each condition in the database has a start date/time and is considered valid until the start date/time of the next value of that condition.

\subsubsection{Query Interface}

Researchers retrieve data from the FishPAS Data Repository using a Query Interface tool. This tool, implemented in Microsoft Access, allows the user to specify criteria to select groups of deployments for further processing. The interface tool lets the user view selected deployment data in a tabular form and export it to generic ASCII files. The interface also communicates with MATLAB (see Section 3.3.3), sending selected data for analysis. 
- Data Selection - The Query Interface consists of an Access form (Figure 3.5) populated with various widgets. List boxes help the user filter data using common criteria, such as study name, deployment location, and experimental treatment, to create a list of desired deployments. The deployments in this list can then be selected for viewing or analysis. Complex queries can be constructed manually in SQL and saved for future use.

- Data Viewing - Selecting an individual deployment displays the metadata information on the form. By selecting certain fields from the deployment data, the user can retrieve additional details about the sample, such as experimental conditions, attributes, and treatment information.

- Data Analysis - When Sensor Fish deployments are selected, the user may analyze their time history data using the Analysis Tools application in MATLAB. (See Section 3.3.3 for details about the Analysis Tools application.)

- Data Export - Selected deployment data may be exported to a comma-delimited ASCII file for use in external applications. An Export utility allows the user to select desired fields for export and define the path and name of the output file.

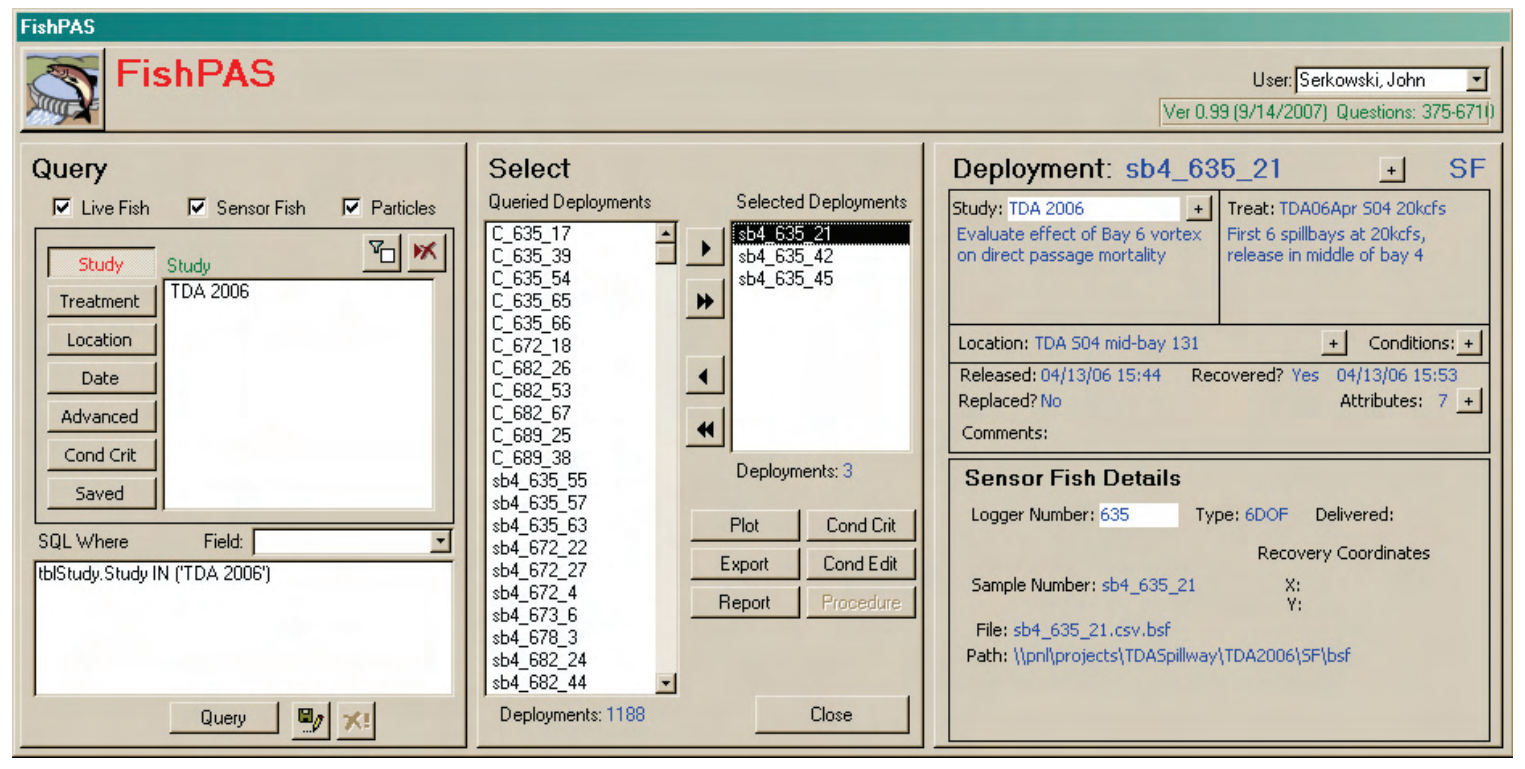

Figure 3.5. FishPAS Query Interface

\subsubsection{Analysis Tools}

The Analysis Tools are incorporated into a graphical user interface that is implemented in MATLAB and consists of a data-plotting screen (Figure 3.6) with widgets for controlling the plot and marking points of interest. The application is initiated by the Query Interface (Section 3.2.3) and runs concurrently with the Access application. The Query Interface provides paths to the binary files that require plotting for the application.

The plotting application allows users to display two variables for up to 20 deployments at a time. The variables are plotted on the left and right $y$-axes, with the $x$-axis always showing time. Each plot axis may be scaled, and each deployment data set may be shifted along the $x$-axis for easier comparison with other deployment data sets. 


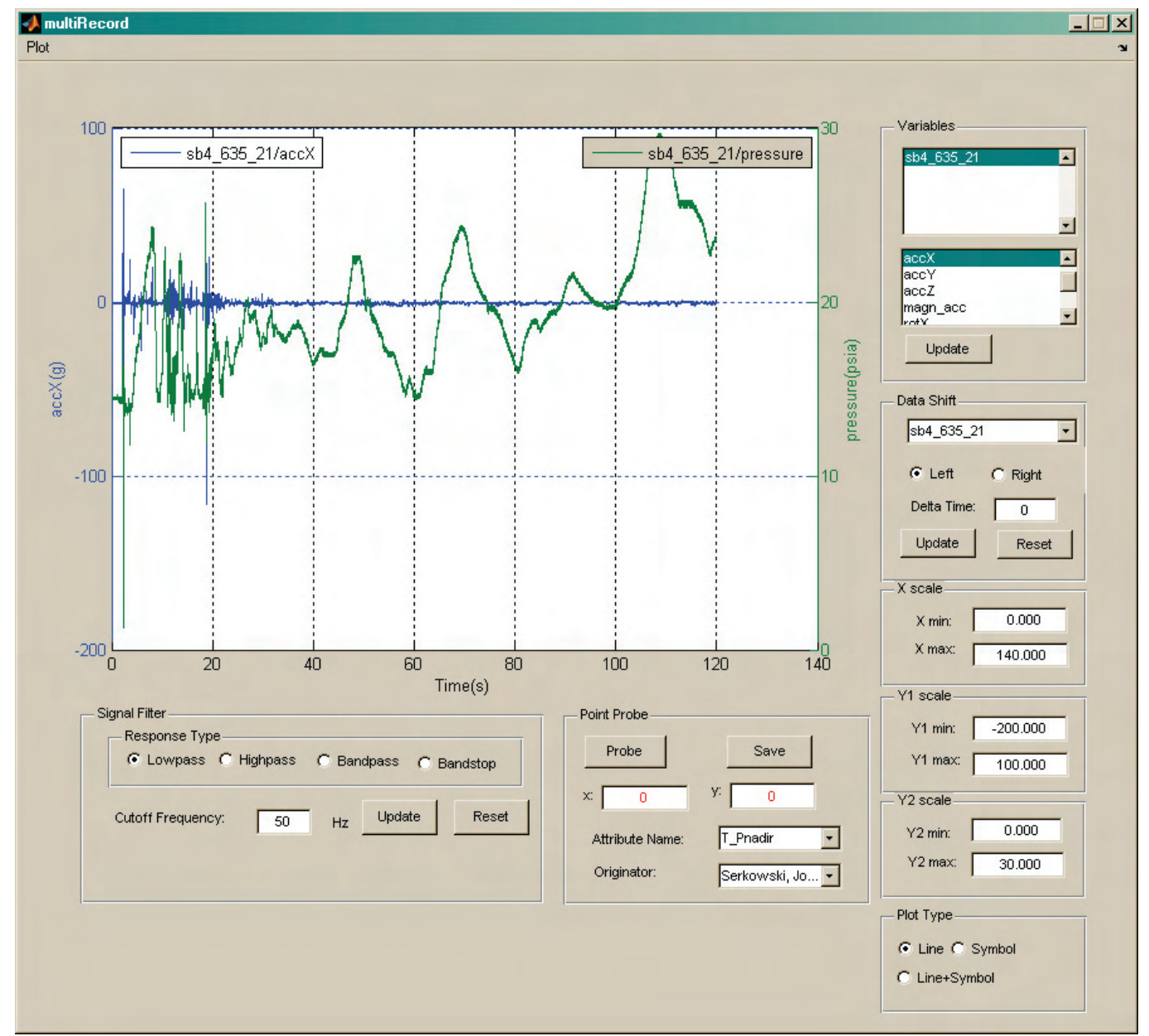

Figure 3.6. MATLAB Analysis Tools Interface

The plotting application also allows users to probe data points on the plot. The results of a probe may be saved back to the FishPAS database as an attribute. To do this, the plot must display only a single deployment and the user must select an attribute name from a dropdown list. When saved, this attribute will be added to the Access database, associated with the selected deployment, and given the time value of the probed point. The communication between MATLAB and Access is performed through Microsoft Automation.

\subsubsection{Hardware and Software Integration}

FishPAS is divided into client and server components operating on the PNNL local area network. For USACE users, another option is to install the complete system in the USACE computer network. The Data Repository resides on one or more server computers with database tables located in a single Access .mdb file and time history data distributed on the hard disks of several file servers.

Each user has a client installation on his or her personal computer, which must be connected to the PNNL intranet. This includes an Access .mdb file containing the Query Interface code with remote links to the Data Repository and the MATLAB Analysis Tools application. The user must have a license for Access to run the Query Interface and have MATLAB Components Runtime (available at no cost) installed. 


\subsection{Data Loading}

Data in the FishPAS repository comes from many sources in a variety of formats. How data are loaded depends on the type and volume of data. Certain subject areas (e.g., Study, Treatment, and Location) contain small numbers of records, and data loading can be managed through manual entry directly into the appropriate tables. Other subject areas (e.g., Deployment and Conditions) may have large numbers of records and, therefore, employ an automated loader to handle data entry. Time history data requires proper formatting of the binary files using conversion programs.

\subsubsection{Loading Methods}

FishPAS uses two primary methods of data loading: manual entry and automated loader programs. Manual data entry entails opening an Access table and typing values into the appropriate fields. This method is used when data are not available in an electronic format and the data volume is small.

Automated loader programs are used when data are available in electronic form, which is usually the case when data volume is large. Loaders used in FishPAS depend on a data input template table (Table 3.2) and some conversion tables (Table 3.3). A template is an empty Access table with a structure that contains fields for all the data to be loaded in a format approximating the source data. A conversion table contains a translation between values used by the data generator, which usually are not standardized, and the FishPAS-accepted value. The input template is filled with the new data, usually by copying and pasting from an Excel spreadsheet, and the automated loader attempts to insert the data into the database. The loader references the conversion tables, which must be correctly populated, checks to validate the completeness and integrity of the data, rejecting records that do not meet requirements, and populates the appropriate database tables. Rejected records are corrected, and loading can be attempted again.

Table 3.2. Data Input Template Tables in FishPAS

\begin{tabular}{c}
\hline Table Name \\
\hline e_tblNewDeployFISH \\
e_tblNewDeploySF \\
e_tblNewDeployPART \\
e_tblNewCondition \\
e_tblNewAttribute \\
\hline
\end{tabular}

Table 3.3. Conversion Tables in FishPAS

\begin{tabular}{c}
\hline Table Name \\
\hline c_tblLocation \\
c_tblMalCause \\
c_tblMalDesig \\
c_tblMeas \\
c_tblProject \\
c_tblSpecies \\
c_tblSurvival \\
c_tblTreatment
\end{tabular}

Because referential integrity is enforced throughout the database (except for the time history files), data must be loaded in a specific order. Lookup lists, which define valid field values, are populated first. Next, tblStudy and tblLocation must be updated, followed by tblTreatment and the condition tables, tblCondition and tblCondMeas. Only after all these subject areas have been loaded can deployment data be inserted. Finally, attribute data can be linked to deployments.

\subsubsection{Lookup Lists}

Lookup list tables generally have a small number of records and are loaded manually using the native Access interface. Valid values are established by the database administrator (see Section 3.5.1). 


\subsubsection{Study, Treatments, and Locations}

The Study, Treatment, and Location subject areas generally contain information that is available prior to the collection of data. This information may be documented in statements of work, test plans, or field notebooks. The tables associated with these data are loaded manually via the native Access interface.

\subsubsection{Conditions}

Experimental conditions data fall into three general categories-Project Operations, CFD Model Setup, and Miscellaneous Conditions. Sources and formats for each of these categories are different, but the volume of data generally requires the use of an automated loader. Due to the variability in the number and types of conditions available from study to study, FishPAS organizes this information in a manner different from that in which it arrives. Most of the data arrive with one row for each date/time (typically at 5-minute or 1-hour intervals) and multiple columns specifying the conditions at each unit or specific location at the dam. However, FishPAS stores each individual measurement as a unique record, so that each row represents a unique date/time, measurement type (e.g., elevation, discharge, blade angle), measurement location (e.g., forebay, Unit 9, Spill Bay 4), and measurement value with units. The input template is structured in the FishPAS format, which means that conditions data need more preprocessing than other types of data in FishPAS. Transformation of the data to the template format can be done manually with copy and paste operations in Excel.

- Project Operations - The vast majority of conditions data consist of project operation and river flow information. These data may be obtained from the USACE in the form of Excel spreadsheets or ASCII files. Sometimes, however, this information arrives with live-fish or Sensor Fish data packages. Regardless of source, the format generally consists of a single record that includes all of the available conditions for a specific date/time. The number of fields in the record varies by project (every project has a different number of locations at which operations are measured) and sometimes within a project (some data packages may only contain a subset of all available operations). To load, the data are transformed into the template format, and the automated loader is run.

- CFD Model Setup - CFD Model Setup is a set of parameters describing the model and particletracking run associated with a particle deployment, excluding the simulated project operations. This may include the name and version of the modeling software, the name of the model result file(s) used, type of turbulence model selected, and parameters to control particle advection. This information is obtained from notes kept by the modeler and are manually entered into the input template (possibly via an Excel spreadsheet) for automated input.

- Miscellaneous Conditions - Other factors that may have a bearing on the experiment (e.g., weather conditions) are obtained from various sources and loaded in the manner of CFD Model Setup conditions.

\subsubsection{Deployments}

The source for deployment information is different for each of the three deployment types-live fish, Sensor Fish, and CFD particles.

- Live Fish Deployments - PNNL receives data for live fish in a Microsoft Excel spreadsheet from the contractor performing the live-fish study, typically Normandeau Associates, Inc. The spreadsheet is reformatted manually to fit a data input template in Access. Reformatting includes rearrangement of columns and substitution of appropriate treatment and condition identifiers. When reformatting is complete, the spreadsheet is pasted into the input template for automated loading. 
- Sensor Fish Deployments - Sensor Fish deployment data are recorded in a Microsoft Excel spreadsheet. As with live-fish data, the spreadsheet is reformatted, transferred to a data input template in Access, and entered into the database via an automated loader.

- CFD Particle Deployments - Particle deployment information is obtained from model run notes. To ease data entry, the necessary information is entered into an Excel spreadsheet, which permits the use of formulas and cell copying. Then, as with the other types of deployment data, a data input template is filled and an automated loader is run.

\subsubsection{Time Histories}

Time history data for Sensor Fish and CFD particle tracks originate as delimited ASCII files. Windows-executable programs are used to convert these files to the proper MATLAB binary format. The files are placed on PNNL intranet fileservers, and their paths are recorded in the appropriate deployment table.

\subsubsection{Attributes}

Attribute data is typically generated automatically during interaction with the Analysis Tools module. Attributes generated outside of FishPAS can be loaded, via a manually loaded input template, with a loader program.

\subsubsection{Miscellaneous Tables}

Supporting tables, such as tblSFCalibration and tblFishPhoto, are updated manually through the native Access interface.

\subsection{Configuration Management}

The quality of FishPAS data analyses depends on the integrity of the data in the database and the validity of the software used to manipulate the data. The tools of configuration management, including validation, testing, change control, and security, are used to ensure that these two components are maintained at the highest levels of accuracy possible, given the constraints of time and budget.

\subsubsection{Roles and Responsibilities}

Participants in the FishPAS database may be classified into the roles defined below. Because FishPAS is a small system, a single person may occupy more than one of these roles:

- client - The client is the person or organization providing or managing the funding for the maintenance of the system. The client's responsibilities include guiding and approving the strategic development of the system and providing sufficient funding to maintain the system according to this document.

- system administrator - The system administrator is a PNNL employee with a thorough knowledge of FishPAS data and software. The system administrator coordinates and documents the configuration management activities described in this section. The administrator also handles other miscellaneous issues concerning the database not addressed here, such as setting up and training new users, maintaining the fileservers, and recovering from system crashes. 
- developer - The developer is a PNNL employee skilled in Microsoft Access and MATLAB programming. This person modifies or adds software as directed by the system administrator.

- data entry technician - The data entry technician is a PNNL employee familiar with Microsoft Access and Excel and performs data entry into the database.

- tester - A tester is a member of any of the other roles, except developer, whose responsibility is to test software after modifications are made. The tester must document the testing and provide the results to the system administrator.

- user - The user is a researcher, or project personnel or manager interested in looking at the data in FishPAS. The user's responsibilities include following the data security procedures outlined in Section 3.5.5 and notifying the system administrator of issues, bugs, and ideas related to FishPAS.

\subsubsection{Data Validation and Verification}

All data loaded into FishPAS undergo some level of data validation (ensuring the data are reasonable and complete) and verification (ensuring the data were transcribed correctly). Some data validation is done by the automated loader programs used to populate the primary tables in the database (see Section 3.4.1). Loader programs may be coded to identify missing data fields, invalid codes, and out-ofrange values. Referential integrity inherent in the structure of the database prevents duplicate keys from being loaded.

Some validation is done on an ad hoc basis when questions about data validity arise. These data sets may then be analyzed for anomalous values using external programs beyond the scope of FishPAS.

Correct entry of data into the database is verified by checking the loaded records against the source document. For manually entered data, a $100 \%$ check is made by someone other than the data entry technician. For automated loader data, only a small sample ( $1 \%-5 \%$ of the data) is verified. If errors are discovered in the automated loader data, the system administrator must determine and fix the cause and reload the data. If a loader error is found, previously loaded data may need to be reloaded as well.

\subsubsection{Baseline Configuration}

Prior to production use, the initial version of FishPAS underwent testing by the primary users. The testers exercised the various capabilities of the software and reported issues and bugs to the system administrator for reconciliation. Testers also determined if all of the system requirements were met satisfactorily. When all issues were resolved, the baseline configuration was established, and version 1.0 of the software was released.

\subsubsection{Change Control}

Modifications to the database structure may be necessary to accommodate some special test conditions or to expand its functionality. In addition, corrections are needed when errors are detected in the data in the database.

Changes made to the system software or to data already loaded into the database must follow an orderly change control process. Because FishPAS is a new system with few users, the process is streamlined for efficiency to encourage development. Its basic foundation is documentation and testing. 
Changes to software may be initiated by anyone in a role associated with FishPAS (see Section 3.5.1) and are passed on to the system administrator. The system administrator confers with the clients and users and, if there is consensus to proceed with the change, instructs the developer to do the coding on a development version of the system. After coding is complete, a tester checks the functionality of the change. If testing is successful, the system administrator archives the production system and moves the development system into production, incrementing the version appropriately. If testing fails, the developer makes corrections and testing is repeated until it succeeds. Through this process, the system administrator maintains a log of the change that should include a description of the modification, how it was implemented, testing results, and the date the new version went into production.

Changes to data in the database also may be initiated by anyone in a role associated with FishPAS and are passed on to the system administrator. The system administrator confers with the generator of the data and, if it is deemed appropriate to correct, instructs a data entry technician to make the correction. The system administrator verifies the correction and documents the circumstances.

\subsubsection{Security}

Data in FishPAS are protected against damage and loss by restricting access and performing regular backups. The main database tables and binary MATLAB files reside on secure fileservers with access restricted to users approved by the system administrator. Access is controlled through Windows filesharing permissions, which require the appropriate intranet username and password.

The system administrator maintains full and incremental backups of data on the servers on external hard drives that are not collocated with the servers. The administrator also maintains a complete archive of all production versions of the FishPAS application.

\subsection{Sample Queries Demonstrating Use of the Database}

For The Dalles Dam spillway, a total of nine direct passage studies have been conducted since 1995. Data collected during the 2001 study were not included because it was a feasibility study and the data were not comparable to those of the other studies. All data sets acquired for the other eight studies were uploaded into the database, with total deployments of 12,342 and 1,050 for live fish and valid Sensor Fish, respectively (Table 3.4). Several corresponding CFD particle runs also were included. The actual use of the database for data access and retrieval is demonstrated by the following two examples.

Table 3.4. Data Input Template Tables in FishPAS

\begin{tabular}{lccc}
\hline Study & $\begin{array}{c}\text { Fish } \\
\text { Released }\end{array}$ & $\begin{array}{c}\text { Valid Sensor } \\
\text { Fish }\end{array}$ & $\begin{array}{c}\text { Particle } \\
\text { Runs }\end{array}$ \\
\hline TDA 1995 & 1226 & N/A & No \\
TDA 2002 August & 949 & 88 & No \\
TDA 2002 May & 1587 & 175 & Yes \\
TDA 2002 October & 1790 & 154 & Yes \\
TDA 2003 & 3156 & 336 & Yes \\
TDA 2004 April & 1212 & 104 & Yes \\
TDA 2004 May & 1340 & 108 & Yes \\
TDA 2006 & 1082 & 85 & No \\
Total & 12342 & 1050 & \\
\hline
\end{tabular}




\subsubsection{Application for Cross-Study Queries}

The purpose of this example is to show cross-study query with a combination of physical release locations and operation parameters. Suppose we need to evaluate the spillway passage conditions at midBay 4, with release elevation lower than $133 \mathrm{ft}$ and tailwater elevation lower than $80 \mathrm{ft}$. The corresponding release details, live fish injuries, and Sensor Fish throughout the years under the defined conditions can be obtained easily as follows:

- Open the query interface and click the Cond Crit button to open the Condition Criteria window (Figure 3.7).

- Define tailwater elevation lower than $80 \mathrm{ft}$ in the Condition Criteria window and save it as a query.

- Select the saved query from the Cond Crit item listed in the Main menu on the left.

- Define the corresponding release location from the Location item listed in the Main menu.

- Select Live Fish, deselect Sensor Fish and Particles, and use the Report function here to export query results in metadata format (Figure 3.8).

- Repeat the process for Sensor Fish by selecting Sensor Fish and deselecting Live Fish and Particles. The results are shown in Table 3.5.

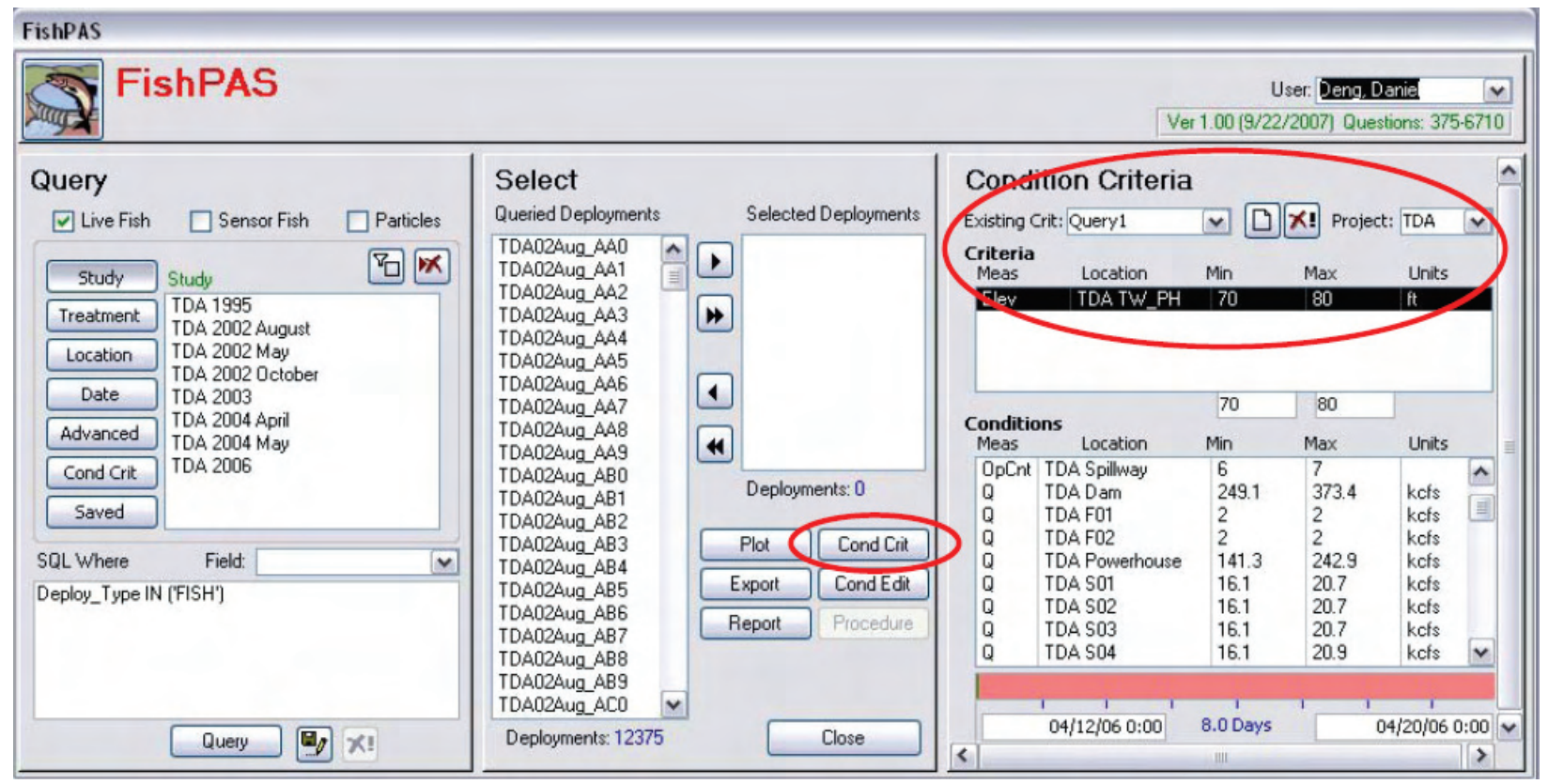

Figure 3.7. Condition Criteria Definition Interface 


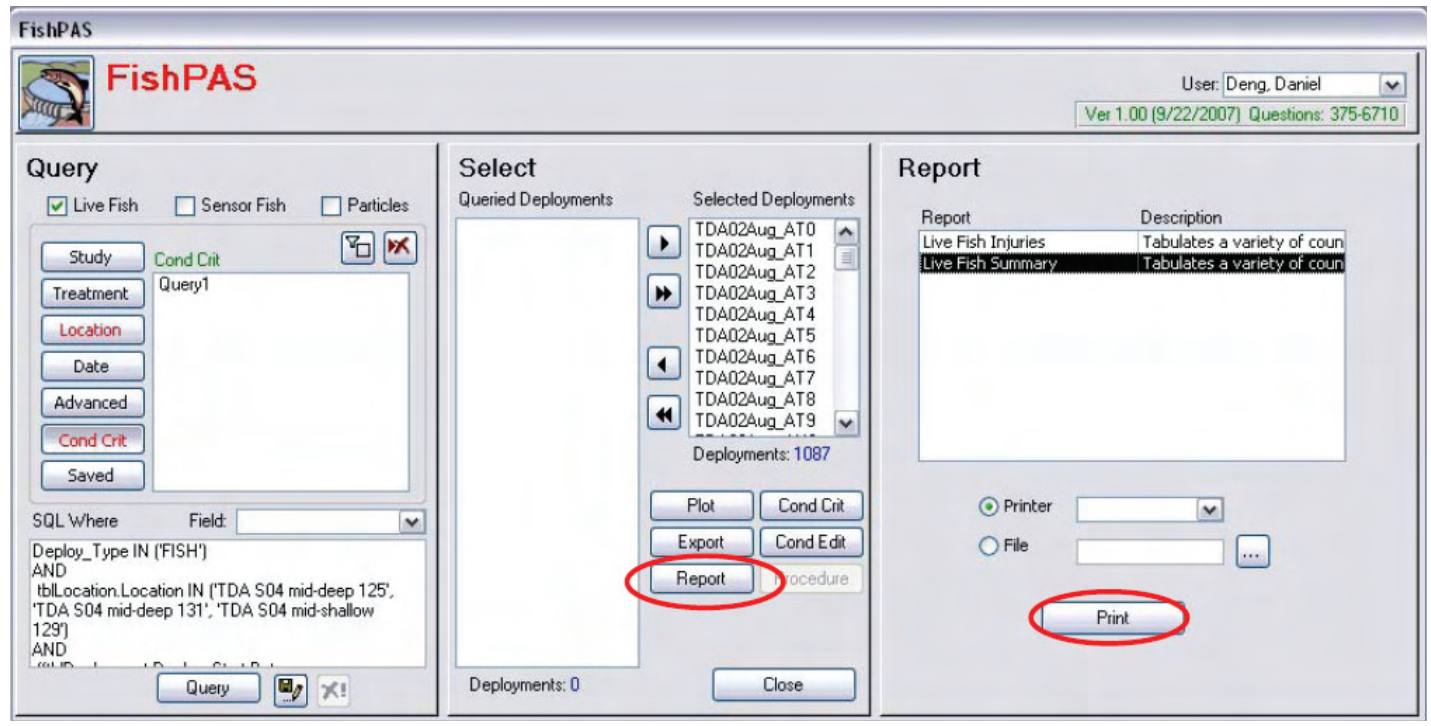

Figure 3.8. FishPAS Reporting and Exporting Interface

Table 3.5. Summary of Query Results for Middle Bay 4 Releases, with Release Elevation Less Than $133 \mathrm{ft}$ and Tailwater Elevation Less Than $80 \mathrm{ft}$

\begin{tabular}{|c|c|c|c|c|c|c|c|}
\hline Studies & $\begin{array}{c}\text { Released } \\
\text { Fish }\end{array}$ & $\begin{array}{l}\text { Alive } \\
\text { After } \\
48 \mathrm{hr}\end{array}$ & $\begin{array}{l}\text { Passage- } \\
\text { Related } \\
\text { Injury }\end{array}$ & $\begin{array}{l}\text { Minor } \\
\text { Injury }\end{array}$ & $\begin{array}{l}\text { Major } \\
\text { Injury }\end{array}$ & $\begin{array}{l}\text { Eye } \\
\text { Injury }\end{array}$ & $\begin{array}{c}\text { Valid Sensor } \\
\text { Fish }\end{array}$ \\
\hline \multicolumn{8}{|l|}{ TDA 1995} \\
\hline TDA 2002 August & 212 & 115 & 15 & 4 & 11 & 2 & 24 \\
\hline TDA 2002 May & 110 & 99 & 8 & 4 & 4 & 5 & 11 \\
\hline TDA 2002 October & 585 & 563 & 19 & 12 & 7 & 5 & 51 \\
\hline TDA 2003 & 68 & 67 & 1 & 0 & 1 & 1 & 7 \\
\hline TDA 2004 April & 112 & 108 & 4 & 2 & 2 & 1 & 11 \\
\hline \multicolumn{8}{|l|}{ TDA 2004 May } \\
\hline TDA 2006 & & & & & & & \\
\hline Total & 1087 & 952 & 47 & 22 & 25 & 14 & 104 \\
\hline
\end{tabular}

\subsubsection{Application for Operations Zone Evaluation}

In 2007, Laurie Ebner proposed an approach to locate low fish injury/mortality zones (so-called sweet spots) for fish passage at The Dalles Dam spillway (USACE Portland District, personal communication). In particular, she suggested the use of discharge-per-bay and tailwater elevation as the starting example. Tailwater elevation was plotted on the $y$-axis and discharge-per-bay was plotted on the $x$-axis. The data were then color-coded to indicate sweet spots and bad zones (Figure 3.9). The two blue zones were identified as sweet spots using water particle-tracking techniques.

Because a complete evaluation requires realistic assumption of fish distribution between different bays, only deployments at Bay 4 are considered for simplicity. Zone 1 is defined as discharge-per-bay between 8 and $12 \mathrm{kcfs}$ and tailwater elevation between 76 and $80 \mathrm{ft}$. In Zone 2, discharge-per-bay ranges from 12 to $18 \mathrm{kcfs}$ and tailwater elevation ranges from 80 to $85 \mathrm{ft}$. Zone 3 is defined as discharge-per-bay between 18 and $24 \mathrm{kcfs}$ and tailwater elevation between 80 and $87 \mathrm{ft}$. Throughout the years, the total numbers of releases at Bay 4 are 710, 954, and 901 in Zones 1-3, respectively, while the associated injury rates are $3.7 \% \pm 0.7 \%, 3.3 \% \pm 0.6 \%$, and $4.1 \% \pm 0.7 \%$, respectively (Figure 3.10 ). 


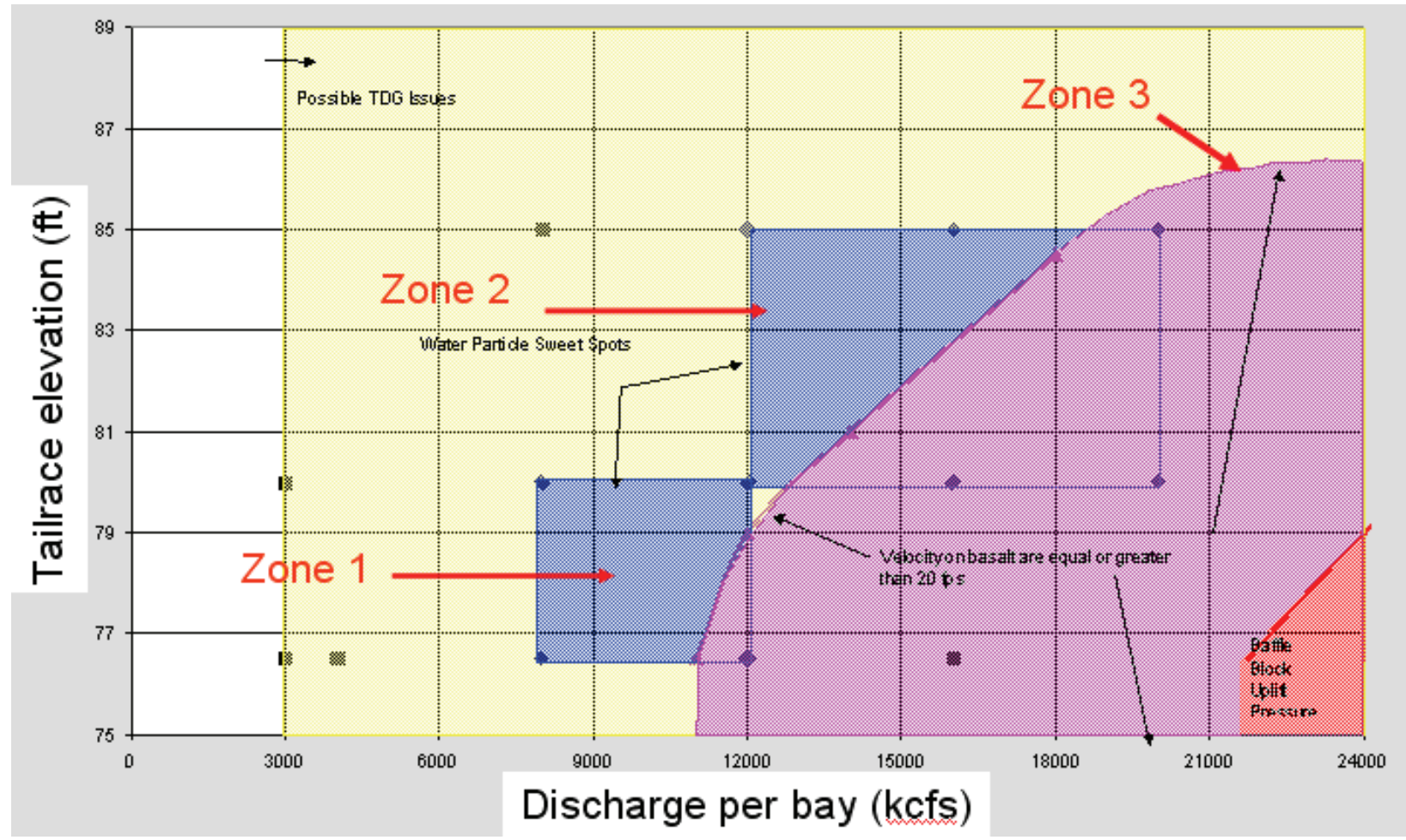

Figure 3.9. Z Zone Definition for Fish Passage Condition Evaluation Using Discharge-Per-Bay and Tailwater Elevation

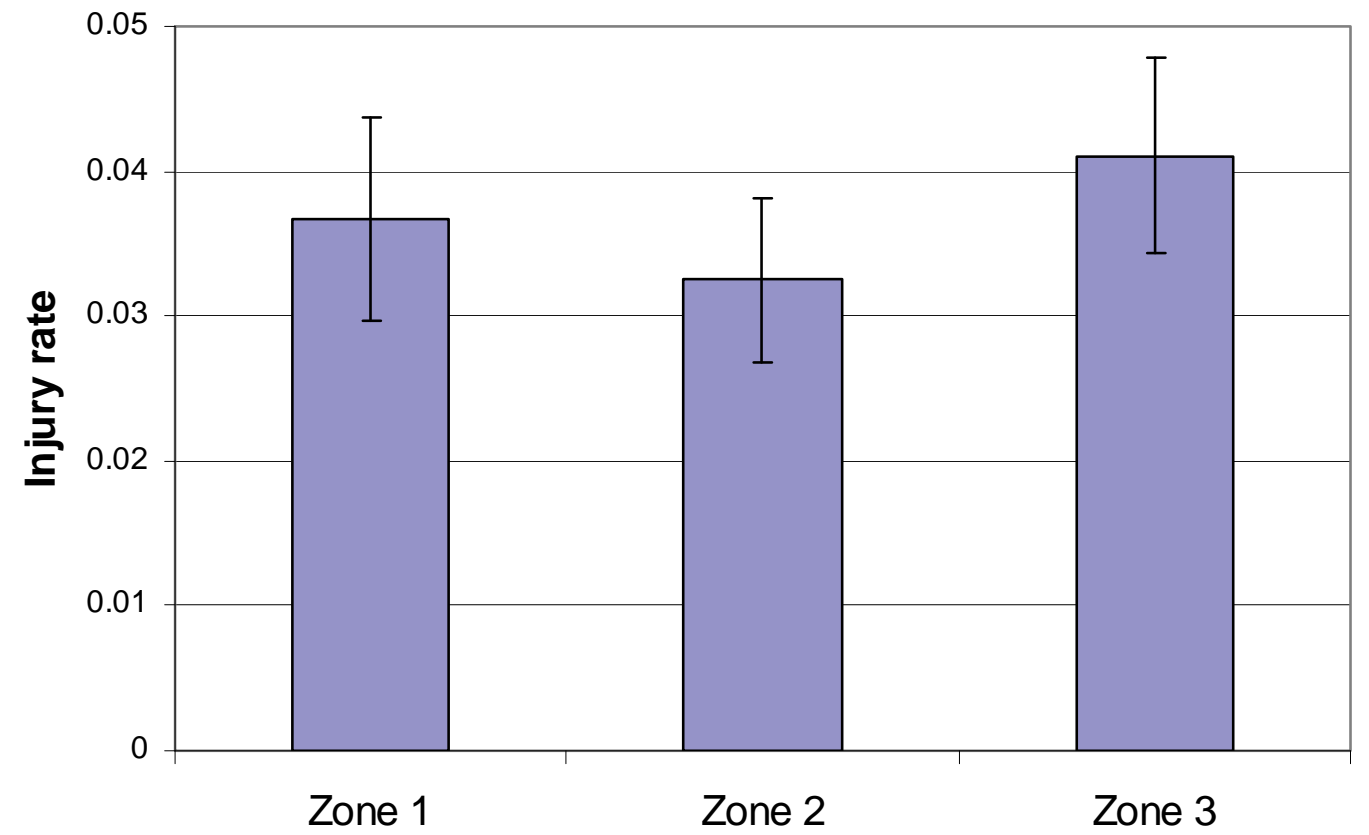

Figure 3.10. Injury Rate Comparison of Fish Released at Bay 4 Under Conditions of the Three Zones 



\subsection{Conclusions and Recommendations}

Release elevations computed, assuming hydrostatic pressure, from Sensor Fish pressure measurements indicated that Sensor Fish might have been released deeper in Bay 4 ( 135 ft) than those in Bay 6 ( 138 ft), with the planned release elevation being $131 \mathrm{ft}$. The accuracy of the hydrostatic condition assumption can be evaluated using flow information from CFD. For the high-volume spills in this study at The Dalles Dam, non-hydrostatic conditions exist near the release pipe exits, and the release elevation error assuming hydrostatic conditions can be up to 2 to $3 \mathrm{ft}$. In addition, Sensor Fish released at Bay 4 measured higher peak pressure in the transition region (from spillway chute to tailwater) than Sensor Fish released at Bay 6, possibly because of the deeper releases at Bay 4 and the existence of a high-pressure zone in the lower water column. However, we have no conclusive evidence with which to determine whether the release pipe exits were actually placed at the target elevations or whether the apparent elevation differences are caused by the non-hydrostatic pressure fields that exist at Bays 4 and 6 .

During passage from the terminus of the injection pipe to the location of stilling basin baffle blocks, Sensor Fish released into Bay 4 showed a higher occurrence of severe events than those released into Bay 6. Overall, for the mid-bay releases in this study, Sensor Fish released from Bay 6 had smoother passage (experienced better quality of flow) than those released from Bay 4 . These findings are consistent with findings of the live fish study in which those fish released from Bay 4 sustained higher injury rates than fish from Bay 6 and the survival rate at Bay 6 was higher than that at Bay 4. In addition, there were significant differences in Sensor Fish measurements between vortex-entrained passage and mid-bay passage. Vortex-entrained Sensor Fish usually experienced higher pressure fluctuations, a larger percentage of severe events, and much more rapid angular velocities. This differs from the findings of the concurrent survival study in which there was almost no injury to the fish released upstream of the vortex. The difference may result from the possibility that the fish were not entrained into the vortex, because the results from a previous study in 2004 showed much higher injury rates for fish thrown into the vortex from the deck. To understand the hydraulic impact of the vortex on juvenile fish, the full study must be completed to collect more samples of vortex-entrained Sensor Fish to compare directly with entrained and non-entrained Sensor Fish released upstream of the vortex under more typical entrainment conditions.

Upon request of the USACE Portland District and Walla Walla District, PNNL convened a meeting to discuss the need and scope of designing and implementing a comprehensive database for the large amount of live fish, Sensor Fish, and CFD particle-tracking data collected during many spillway fish passage studies in the Columbia Basin. The spillway at The Dalles Dam was selected as a case study to demonstrate the functionality of a comprehensive database containing Sensor Fish, live fish, and CFD simulation data, because The Dalles Dam spillway is one of the most complex in the Pacific Northwest region and has the most comprehensive data set. The resulting database application is Fish Passage Analysis System (FishPAS).

FishPAS was configured to synthesize Sensor Fish data, live fish data, CFD particle-tracking data, and operations data for the assessment of fish passage conditions at turbines, spillways, and bypass facilities for all hydroelectric projects while being focused on studies conducted at The Dalles Dam spillway. The Dalles Dam spillway was used as a case study to demonstrate the functionality of the database. The database was implemented using Microsoft Access 2003, a widely available relational database management system, and MathWorks MATLAB, a scientific analysis application. The data storage structure incorporates handling of passage condition data collected using various Sensor Fish 
generations, live fish data, and CFD particle-tracking data. The database interface provides researchers versatility in how the data can be queried and retrieved for different dam operation conditions.

In September 2007, another meeting was held to review the results of the case study. The USACE participants recommended that the database be extended to system-wide use. The suggested extension is targeted on work planned for John Day Dam in 2008 and other future studies in addition to existing balloon-tagged live fish data sets and the 6DOF Sensor Fish data sets. For future studies, after Sensor Fish data are acquired, processed initially, and checked for quality, they will then be included in the database with detailed operations data. PNNL will interact with the assigned USACE point of contact and Normandeau Associates, Inc., to arrange for transfer of live-fish injury and mortality data. The USACE will then interact with PNNL to develop scope and objectives for synthesis and analysis using the updated database. 


\subsection{References}

Carlson TJ and JP Duncan. 2003. Evolution of the Sensor Fish Device for Measuring Physical Conditions in Severe Hydraulic Environments. PNNL-15708, Pacific Northwest National Laboratory, Richland, Washington.

Carlson TJ and JP Duncan. 2004. Characterization of Spillway Passage Conditions at Ice Harbor Dam, Snake River, Washington, 2003. PNWD-3462, Battelle-Pacific Northwest Division, Richland, Washington.

Carlson TJ, JP Duncan, MC Richmond, JA Serkowski, and Z Deng. 2006. Characterization of Spillway Passage Conditions at Lower Monumental Dam, Snake River, Washington, 2005. PNWD-3662, BattellePacific Northwest Division, Richland, Washington.

Deng Z, MC Richmond, CS Simmons, and TJ Carlson. 2004. Six Degrees of Freedom Sensor Fish Design: Governing Equations and Motion Modeling. PNNL-14779, Pacific Northwest National Laboratory, Richland, Washington.

Deng Z, GR Guensch, CA McKinstry, RP Mueller, and MC Richmond. 2005. "Evaluation of FishInjury Mechanisms During Exposure to Turbulent Shear Flow.” Canadian Journal of Fisheries and Aquatic Sciences 62(7):1513-1522.

Deng Z, MC Richmond, and TJ Carlson. 2006. Characterization of The Dalles Dam Spillbay 6 Vortex Using Surface Entrained Sensor Fish Device: Preliminary Report. PNNL-15883, Pacific Northwest National Laboratory, Richland, Washington.

Deng Z, TJ Carlson, JP Duncan, and MC Richmond. 2007. “Applications of the Sensor Fish Technology." Hydro Review 26(5):34-41.

Dauble DD, Z Deng, MC Richmond, RA Moursund, TJ Carlson, and CL Rakowski. 007. Biological Assessment of the New Turbine at Wanapum Dam, 2005. PNNL-16682, Pacific Northwest National Laboratory, Richland, Washington.

Efron B and RJ Tibshirani. 1993. An Introduction to the Bootstrap. Chapman \& Hall, New York.

IEEE. 2006. IEEE Standard for Developing a Software Project Life Cycle Process. IEEE 1074-2006, Institute of Electrical and Electronics Engineers, Piscataway, New Jersey.

Normandeau Associates, Inc. and JR Skalski. 2006. Juvenile Salmonid Survival/Condition Upon Passing the Dalles Dam Spillbays 4 and 6 and a Vortex at Higher Spill Levels. Prepared for the U.S. Army Corps of Engineers, Portland District, Portland, Oregon.

Normandeau Associates, Inc. and JR Skalski. 2005. Evaluation of Training Wall on Fish Condition at The Dalles Dam Spillway, Columbia River, 2004. Prepared for the U.S. Army Corps of Engineers, Portland District, Portland, Oregon. 
Richmond MC, TJ Carlson, JA Serkowski, CB Cook, JP Duncan, and WA Perkins. 2007.

Characterizing the Fish Passage Environment at The Dalles Dam Spillway: 2001-2004. PNNL-16521, Pacific Northwest National Laboratory, Richland, Washington. 


\section{Appendix}

\section{Guidelines for Major and Minor Injury Classifications at} Turb'N Tag Studies 



\section{Appendix}

\section{Guidelines for Major and Minor Injury Classifications at Turb'N Tag Studies}

- A fish with only LOE is classified as major if the fish dies within 1 hour. If it survives or dies beyond 1 hour, it is classified as minor.

- A fish with no visible external or internal maladies is classified as a passage-related major injury if the fish dies within 1 hour. If it dies beyond 1 hour, it is classified as a non-passage-related minor injury.

- Any minor injury that leads to death within 1 hour is classified as a major injury. If it lives or dies after 1 hour, it remains a minor injury.

- Hemorrhaged eye: minor if less than 50\%; major if 50\% or more.

- Deformed pupil(s) are a major injury.

- Bulged eye is major unless one eye is only slightly bulged; minor if slight.

- Bruises are size-dependent; considered major if $10 \%$ or more of fish body per side; otherwise minor.

- Operculum tear at dorsal insertion is major if it is $5 \mathrm{~mm}$ or greater; otherwise minor.

- Operculum folded under or torn off is a major injury.

- Scale loss is major if $20 \%$ or more of fish per side; otherwise minor.

- Scraping (damage to epidermis) is major if $10 \%$ or more per side of fish; otherwise minor.

- Cuts and lacerations are generally classified as major injuries. Small flaps of skin or skinned up snouts are minor.

- Internal hemorrhage or rupture of kidney, heart, or other internal organs that results in death at 1 to 48 hours is a major injury.

- Multiple injuries: use the worst injury. 